Staff Working Paper/Document de travail du personnel 2020-2

Last updated: January 27, 2020

\title{
Social Learning and Monetary Policy at the Effective Lower Bound
}

by Jasmina Arifovic ${ }^{1}$, Alex Grimaud ${ }^{2}$, Isabelle Salle ${ }^{3}$ and Gauthier Vermandel $^{4}$

\footnotetext{
${ }^{1}$ Department of Economics

Simon Fraser University

Burnaby, British Columbia, Canada

arifovic@sfu.ca

2 Department of Economics and Finance Catholic University of Milan, IT, and

Amsterdam School of Economics, University of Amsterdam, NL a.b.p.grimaud@uva.nl

${ }^{3}$ Financial Markets Department

Bank of Canada

Ottawa, Ontario, Canada K1A 0G9

ISalle@bank-banque-canada.ca

${ }^{4}$ Paris-Dauphine University, FR and

France Stratégie, FR

gauthier@vermandel.fr
}

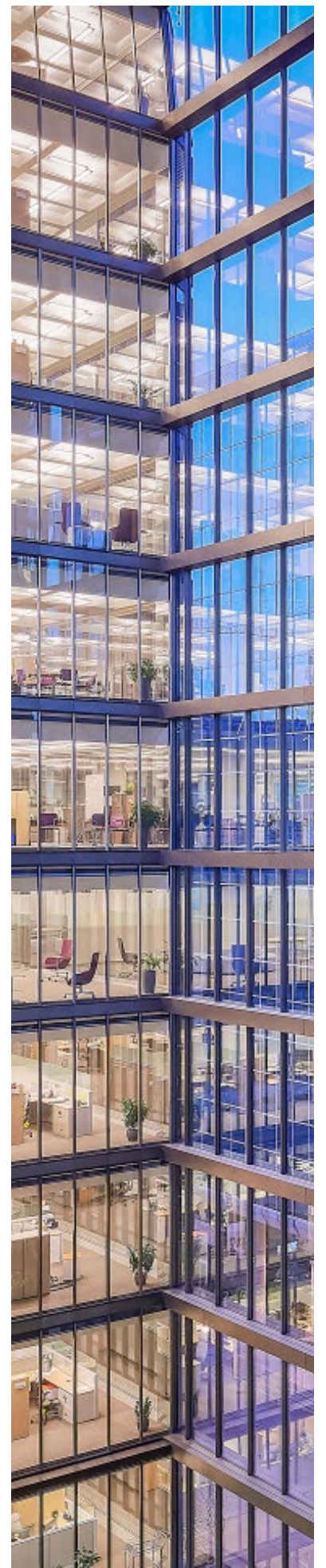

Bank of Canada staff working papers provide a forum for staff to publish work-in-progress research independently from the Bank's Governing Council. This work may support or challenge prevailing policy orthodoxy. Therefore, the views expressed in this note are solely those of the authors and may differ from official Bank of Canada views. No responsibility for them should be attributed to the Bank. 


\section{Acknowledgements}

The present work has benefited from fruitful discussions and helpful suggestions from Jim Bullard, Ben Craig, Pablo Cuba-Borda, George Evans, Jordi Galí, Tomáš Holub, Cars Hommes, Seppo Honkapohja, Robert Kollmann, Douglas Laxton, Albert Marcet, Domenico Massaro, Bruce McGough, Athanasios Orphanides, Bruce Preston, Jonathan Witmer and Raf Wouters. We also thank the participants of the Workshop on Adaptive Learning on May 7-8, 2018, in Bilbao; the 2018 EEA-ESEM meeting in Cologne; the seminar at the Bank of Latvia on March 23, 2019; the workshop on Expectations in Dynamic Macroeconomic Models at the 2019 GSE Summer Forum in Barcelona; the 2019 Computing Economics and Finance Conference in Ottawa; the 15th Dynare Conference in Lausanne; the 22nd Central Bank Macroeconomic Modeling Workshop in Dilijan, in particular our discussant Junior Maih; the internal seminar at the Bank of Canada on September 22, 2019; and the 2019 SNB research conference in Zurich. This work has received funding from the European Union Horizon 2020 research and innovation program under the Marie Sklodowska-Curie grant agreement No. 721846, "Expectations and Social Influence Dynamics in Economics." We thank William Ho for helpful research assistance. None of the above are responsible for potential errors in the paper. The views expressed in the paper are those of the authors and do not necessarily reflect those of the Bank of Canada. 


\section{Abstract}

The first contribution of this paper is to develop a model that jointly accounts for the missing disinflation in the wake of the Great Recession and the subsequently observed inflation-less recovery. The key mechanism works through heterogeneous expectations that may durably lose their anchorage to the central bank (CB)'s target and coordinate on particularly persistent below-target paths. We jointly estimate the structural and the learning parameters of the model by matching moments from both macroeconomic and Survey of Professional Forecasters data. The welfare cost associated with those dynamics may be reduced if the $C B$ communicates to the agents its target or its own inflation forecasts, as communication helps anchor expectations at the target. However, the CB may lose its credibility whenever its announcements become decoupled from actual inflation, for instance in the face of large and unexpected shocks.

Bank topics: Monetary policy; Monetary policy communication; Credibility; Central bank research; Economic models; Business fluctuations and cycles

JEL codes: C82, E32, E52, E70 


\section{Introduction}

The Great Recession in the US and Europe and the ensuing monetary policy reactions have given way to a 'new normal' in economic conditions: interest rates have remained below target. This situation is particularly acute in Europe, where interest rates are still at the effective lower bound (ELB). Yet, no substantial changes in the price levels have been recorded, neither in the wake of the downturn - despite the severity of the recession - nor along the recent output growth episode, which then resembles an inflation-less recovery. Meanwhile, inflation expectations have remained consistently below target, as depicted in Figure 1, which puts at risk the long-run anchorage of expectations. This risk is exacerbated by the structural decline in natural interest rates observed over the last decades, which exerts further downward pressure on inflation expectations (Mertens \& Williams 2019). Low inflationary pressures have now pushed a number of major central banks (CBs) to further ease monetary policy.

This low-inflation narrative is hard to unfold within the standard macroeconomic model - namely the New Keynesian (NK) class of models - for at least two reasons.
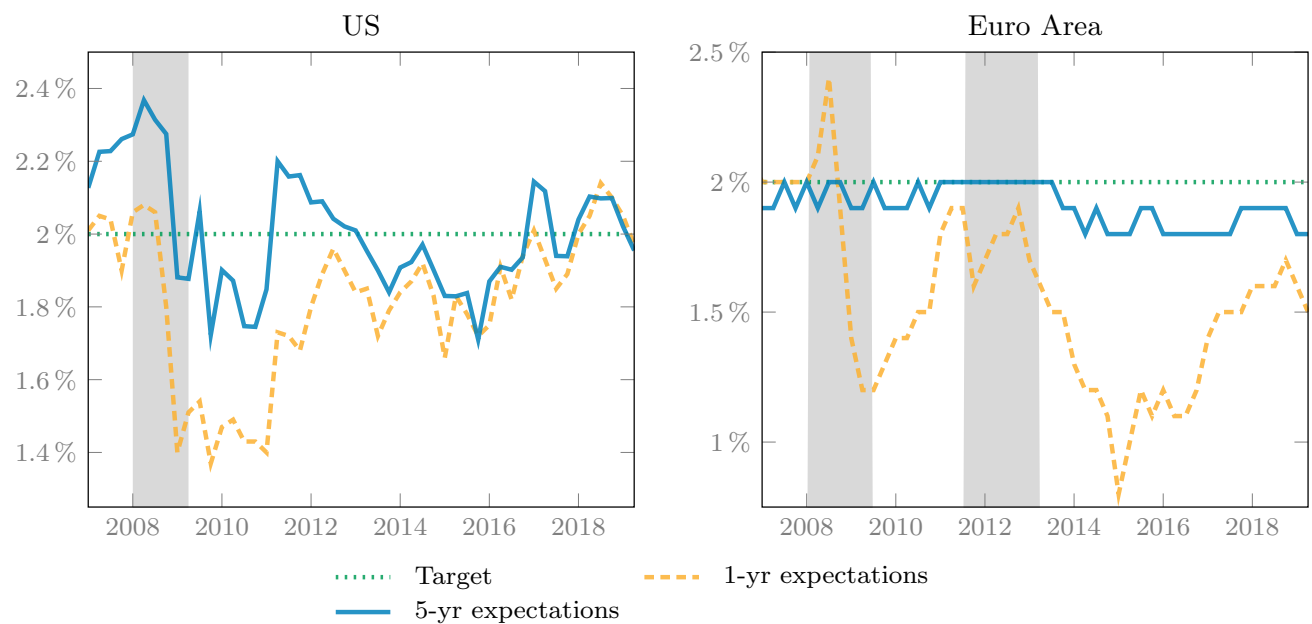

Notes: The shaded areas represent the recessions as dated by the National Bureau of Economic Research (NBER) and Centre for Economic Policy Research (CEPR), and the green dashed lines the inflation targets. Data are from the Survey of Professional Forecasters (SPF) and the European Central Bank (ECB).

Figure 1: Inflation expectations in the US and Euro Area 2008-2019 


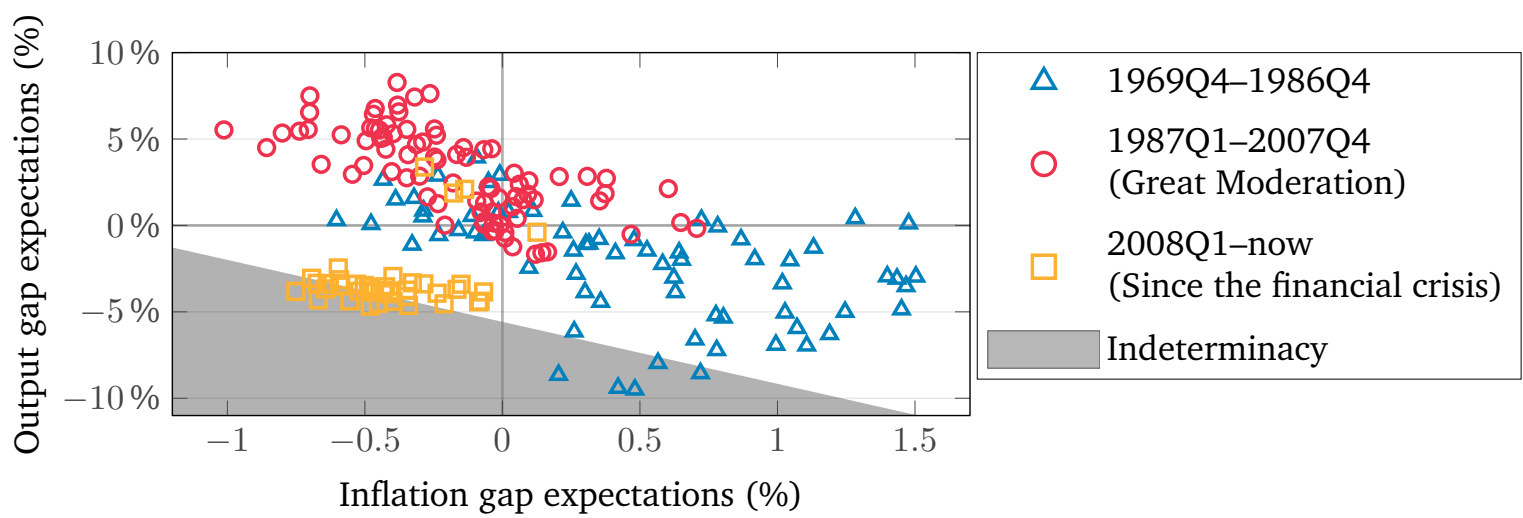

Notes: The shaded area denotes the region of the state space that violates the determinacy condition under rational expectations (REs) in the sense of Blanchard \& Kahn (1980) and leads to diverging deflationary spirals under recursive learning. In contrast, the white area denotes the region of the state space that is determinate, which is also the basin of attraction of the target under recursive learning. Data on expectations are taken from the Survey of Professional Forecasters. The output gap is computed using a linear trend. Calibration of the New Keynesian model is taken from Galí (2015).

Figure 2: (Ir)relevance of the New Keynesian model with rational expectations since the 'new normal'

First, zero interest rates generate implausible macroeconomic dynamics in those models. Under rational expectations (RE), the dynamics are indeterminate at the ELB (Benhabib et al. 2001), which implies excess volatility in inflation that is clearly at odds with the recent experience. This puzzle is clearly visible from survey data, which have been lying in the indeterminacy region of the inflation-output state space since the financial crisis, as depicted in Figure 2. Replacing RE by boundedly rational and learning agents induces diverging deflationary spirals at the ELB, which does not match the current situation either (Evans et al. 2008).

Second, the standard assumption of complete information and common beliefs leaves little room for expectations to be persistently off the target and play any autonomous role in driving business cycles. In those models, recessive episodes are typically generated by exogenous and persistent technology or financial shocks. ${ }^{1}$ Not only does this conception of expectations conflict with the empirical evidence of unanchored and

\footnotetext{
${ }^{1}$ There are some recent exceptions, e.g. Angeletos et al. (2018), who investigate the role of strategic uncertainty in the presence of heterogeneous information within a general equilibrium model. However, those authors use a real business cycle (RBC) model, which implies that monetary policy is left out.
} 
dispersed forecasts that has been found in survey data, ${ }^{2}$ but it also does not leave any room for monetary policy to influence or coordinate private expectations through communication.

Therefore, the main contribution of this paper is to address these challenges by developing a model in which time-varying and persistent heterogeneity in expectations endogenously produces ELB dynamics so as to account for the recent economic experience. The use of heterogeneity and learning in agents' expectations is not anecdotal given the large literature documenting deviations from RE in real-world expectations and, in particular, pervasive and time-varying heterogeneity. ${ }^{3}$ Heterogeneity in expectations also poses a challenge to the $\mathrm{CB}$ when attempting to coordinate the private sector on the desirable inflation target. Moreover, forecast dispersion has been directly related to macroeconomic uncertainty (Rossi \& Sekhposyan 2015) and has been proven to induce adverse effects on the economy (Jo \& Sekkel 2019).

Specifically, we develop a micro-founded NK model featuring inflation and output dynamics to which we add a parsimonious two-operator evolutionary learning process that specifies the dynamics of expectations and nests the RE homogeneous agent benchmark. This latter feature, together with the sole use of white noise fundamental shocks, isolates learning as the only possible source of persistence in the endogenous variables and allows us to identify the amplifier role of expectations in driving business cycles. In our model, agents form beliefs about the long-run values of inflation and output. This easily translates into the issue of expectation anchorage. Our choice of a social learning

\footnotetext{
${ }^{2}$ For instance, using survey data, Coibion et al. (2019) show that more than half of the firms and households typically do not know the value of the Fed inflation target, while only $20 \%$ of them pick the correct answer. Using the Michigan Survey of Consumers, Coibion et al. (2018) find that one-yearahead households' expectations are on average 1.5 percentage points (p.p.) above the $2 \%$ target of the Fed. The cross-sectional dispersion is also large, up to 3 p.p. in March 2018. Furthermore, Coibion et al. (2018) show that making information salient, notably by providing announcements that are sufficiently clear for the general population, allows the CB to curb inflation expectations and therefore real interest rates.

${ }^{3}$ On deviations from RE in general, we refer to, inter alia, Mankiw et al. (2003), Del Negro \& Eusepi (2011) and Branch (2004). On heterogeneity in particular, see, e.g., Hommes (2011) for evidence using lab forecasting experiments; Mankiw et al. (2003) in survey data from professional forecasters; and Cavallo et al. (2017) from households.
} 
(SL) process is motivated by the parsimony of this class of learning models, their ability to match experimental findings and the evolutionary role of heterogeneity in the adaptation of the agents (Arifovic \& Ledyard 2012). In these models, agents collectively adapt to an ever-changing environment in which their own expectations contribute to shape the macroeconomic variables that they are trying to forecast. Specifically, SL agents dynamically improve their individual forecast strategy through stochastic exploration and imitation of other agents with better historical accuracy in their forecasts. Per consequence, optimistic or pessimistic agents' strategies drive aggregate expectations during booms or busts thanks to their improved accuracy. This feature is well suited to self-referential economic systems such as standard macroeconomic models. SL expectations also find an intuitive interpretation that is reminiscent of the idea of epidemiological expectations where 'expert forecasts' only gradually diffuse across the entire population (Carroll 2003).

In a novel effort within the related literature, ${ }^{4}$ we take our stylized model to the data and show that it is able to jointly replicate ten salient business cycle moments from the Survey of Professional Forecasters (SPF) and the main US macroeconomic time series, including the frequency of ELB episodes, major dimensions of heterogeneity in expectations and a substantial share of the persistence in output and inflation data.

This empirical exercise is already a remarkable result given the parsimony of the model. Yet, it is important to note that we do not aim to contrast the matching abilities of the SL model regarding macroeconomic time series with those of an RE counterpart. For a fair comparison, the SL model would need to compete with an RE version of the model with sunspot dynamics at the ELB. While certainly a needed exercise, it is beyond the scope of the present paper. What our empirical exercise

\footnotetext{
${ }^{4}$ Del Negro \& Eusepi (2011) attempt to replicate expectation data with RE models. Milani (2007) fits an adaptive learning NK model to macroeconomic time series only. Closer to our contribution, Slobodyan \& Wouters $(2012 \underline{a}, b)$ fully estimate an NK model on both macroeconomic and expectations times series. However, the authors use exogenous autocorrelated shocks on expectations to reproduce the observed persistence in the data.
} 
does add to the literature is $(i)$ an estimation routine of a non-linear model under heterogeneous expectations and $(i i)$ an empirical discipline device to learning models by offering estimated values to the learning parameters for which there are no observable counterparts. $^{5}$

A second major contribution is to show that our model endogenously produces stable dynamics at the ELB. Those stable dynamics correspond to inflation-less recoveries, i.e. inflation persists for an extended period of time below its target, the ELB binds, but output expands. ${ }^{6}$ Such a configuration corresponds to the recent economic experience. This means that our simple framework can jointly account for the missing deflation in the wake of the crisis and the missing inflation in the wake of the recovery. In our model, recessive dynamics arise endogenously when agents coordinate on pessimistic expectations following a series of adverse but non-autocorrelated shocks. From there, the transition back to the target can be particularly long if expectations have become unanchored and, per their self-fulfilling nature, nurture the bust. Hence, we offer a reading of the recent economic experience as a long-lasting coordination of agents on pessimistic expectations rather than as the result of persistent and exogenous financial or technological shocks. The forces underlying our narrative are reminiscent of the earlier Keynesian concept of animal spirits.

Furthermore, we introduce central bank communication as a welfare-enhancing tool to coordinate heterogeneous expectations. Given that our model nests the RE homogeneous-agent benchmark, we interpret the dispersion of expectations as a friction and quantify the ensuing welfare loss with respect to the RE outcome. We find that heterogeneous expectations entail a consumption loss of almost $3.3 \%$ with respect to the RE allocation. This highlights how crucial heterogeneity in expectations is with

\footnotetext{
${ }^{5}$ The usual practice in the learning literature is to rely on calibrated models with no quantitative assessment of their empirical relevance in terms of replication of business cycle moments.

${ }^{6}$ The following result would also hold in the presence of unconventional monetary policy captured by a shadow interest rate falling beyond zero and a larger output expansion.
} 
respect to the representative agent construction of the standard NK model. From there, a natural follow-up analysis is to introduce an additional monetary policy instrument next to the interest rate, namely $\mathrm{CB}$ communication, and investigate whether it may offset the costs of forecast dispersion and deliver the RE representative agent benchmark. To address this question, we exploit the flexibility of our parsimonious learning model, which enables us to integrate $\mathrm{CB}$ communication into the learning process of the agents. We show that announcing the inflation target or inflation forecasts may help enforce coordination of agents' expectations on the target. As coordination on pessimistic outlooks is the source of the aggregate propagation of shocks in our model, CB communication reduces the occurrence as well as the severity of ELB episodes and cuts the welfare loss due to heterogeneous expectations, which brings the CB closer to, but nonetheless below, the RE outcome. However, in the face of large unexpected shocks, the CB may lose credibility whenever the announcements become decoupled from the actual realizations of inflation.

Related literature Our treatment of communication adds to the existing literature on communication under learning by modeling endogenous credibility. ${ }^{7}$ The closest to our concept of endogenous credibility is the work by Hommes \& Lustenhouwer (2019), who derive the stability conditions of the targeted equilibrium in an NK model with ELB where agents' expectations switch to follow past inflation, should the target be missed.

A rapidly expanding literature on Heterogeneous Agent New Keynesian (HANK) models investigate the consequences of heterogeneous agents on monetary policy design (see, for a recent account, Galí (2018) and the references herein). While this literature is mostly concerned with the aggregate effects of idiosyncratic shocks on households'

\footnotetext{
${ }^{7}$ The learning literature usually concludes that communication is stabilizing under learning in models where communication imposes model-consistent restrictions on the forecasting model used by the learning agents; see e.g. Eusepi \& Preston (2010). However, a crucial assumption in these models is that communication is credible.
} 
income, we model heterogeneity in expectations. Their implementation of heterogeneity also results in models that are considerably more complicated than ours, as it requires, among other challenges, keeping track of the wealth distribution over time.

In addition, our work substantially differs from New Keynesian models with multiple equilibria where a liquidity trap episode is understood as an exogenously driven regime switch from the targeted equilibrium to the deflationary steady-state (Aruoba et al. 2017, Jarociński \& Maćkowiak 2018). While we also aim to explain the persistent slump after the Great Recession, we do so by using a learning model under which the basin of attraction of the target is larger than the determinacy region under RE. In the context of our model, expectations formed by the learning agents may occasionally visit regions of that basin from where the transition back on target is particularly slow. Those shifts in expectations arise because the interplay between SL and fundamental shocks may cause agents to 'pick up' a downward trend in inflation and output gaps following a series of bad shocks, rather than as a result of the use of sunspots as expectation coordination devices. Schmitt-Grohé \& Uribe (2017) consider exogenous confidencedriven rather than sunspot-driven regime changes but do not model the unanchoring process of expectations from the target. Furthermore, we add to this literature the treatment of communication and endogenous credibility of the CB's announcements.

We borrow from Arifovic et al. $(2013,2018)$ a similar SL mechanism to model expectations within a NK model. However, our present work differs along important dimensions. Among others, those two contributions study the long-run stability of the model as defined by the asymptotic convergence towards a particular equilibrium under $\mathrm{SL}$, while we focus on the short-term fluctuations arising from the interplay between fundamental shocks and learning dynamics. None of those models are taken to the data, and only Arifovic et al. (2018) introduce the ELB but use exogenous shocks to trigger liquidity trap episodes. Those authors interpret such episodes as the anchoring of expectations on the low inflation steady-state, which is not, as explained previously, 
the mechanism generating liquidity traps in this paper. In other words, in our model, we do not contemplate the possibility of the deflationary steady-state to be an attractor of the learning process. While Arifovic et al. (2018) show that the low inflation steadystate may be a stable attractor of their SL mechanism, our implementation of the fitness function and our empirical calibration differ from theirs, which does not allow us to generalize their result to our setup. Furthermore, Arifovic et al. (2013, 2018) do not consider $\mathrm{CB}$ communication and do not measure the welfare implications of that departure from RE.

The rest of the paper proceeds as follows. In Section 2, we develop the model; the estimation is presented in Section 3; the dynamic properties of the model are analyzed in Section 4; Section 5 discusses the effects of CB communication; and Section 6 concludes.

\section{The model}

We first describe the building blocks of the model, then we discuss the solution under the RE benchmark and finally explain our implementation under SL.

\subsection{A piecewise linear New Keynesian model}

Our model builds on the workhorse three-equation NK model developed by, inter alia, Woodford (2003). The three equations describe aggregate demand, aggregate supply and monetary policy. All variables below are expressed in deviation from their steadystate level as targeted by the CB.

Aggregate demand is described by the IS curve:

$$
\widehat{y}_{t}=\mathbb{E}_{j, t}^{*} \widehat{y}_{t+1}-\sigma^{-1}\left(\widehat{\imath}_{t}-\mathbb{E}_{j, t}^{*} \widehat{\pi}_{t+1}\right)+\widehat{g}_{t}
$$

where $\widehat{y}_{t}$ is the output gap, $\widehat{\imath}_{t}$ the nominal interest rate set by the $\mathrm{CB}, \widehat{\pi}_{t}$ the deviation 
of the inflation rate from the target (hence, $\widehat{\imath}_{t}-\mathbb{E}_{t} \widehat{\pi}_{t+1}$ represents the real interest rate), $\sigma>0$ the inter-temporal elasticity of substitution of consumption (based on a CRRA utility function), and $\mathbb{E}_{j, t}^{*}$ the (possibly boundedly rational) expectation operator based on information available at time $t$. The subscript $j$ is introduced to suggest the possibility of heterogeneous expectations, where each agent-type $j=1, \ldots, N$ forms her own expectation (with $N$ the number of agent-types). ${ }^{8} \widehat{g}$ is an exogenous real disturbance.

The supply side is summarized by the forward-looking NK Phillips Curve:

$$
\widehat{\pi}_{t}=\beta \mathbb{E}_{j, t}^{*} \widehat{\pi}_{t+1}+\kappa \widehat{y}_{t}+\widehat{u}_{t}
$$

where $0<\beta<1$ represents the discount factor, $\kappa>0$ a composite parameter capturing the sensitivity of inflation to the output gap and $\widehat{u_{t}}$ an exogenous cost-push shock.

In the RE literature, the shocks $\widehat{g}$ and $\widehat{u}$ are usually assumed to be $\mathrm{AR}(1)$ processes:

$$
\begin{aligned}
& \widehat{g}_{t}=\rho^{g} \widehat{g}_{t-1}+\varepsilon_{t}^{g}, \\
& \widehat{u}_{t}=\rho^{u} \widehat{u}_{t-1}+\varepsilon_{t}^{u},
\end{aligned}
$$

where $0 \leq \rho^{u}, \rho^{g}<1$ measure the persistence of the shocks and $\varepsilon^{g}$, $\varepsilon^{u}$ are i.i.d. with respective standard deviations $\sigma^{g}$ and $\sigma^{u}$.

Monetary policy implements a flexible inflation-targeting regime subject to the ELB constraint, which results in the following non-linear forward-looking Taylor rule:

$$
\widehat{\imath_{t}}=\max \left\{-\bar{r} ; \phi^{\pi} \mathbb{E}_{j, t}^{*} \widehat{\pi}_{t+1}+\phi^{y} \mathbb{E}_{j, t}^{*} \widehat{y}_{t+1}\right\},
$$

\footnotetext{
${ }^{8} \mathrm{We}$ follow here most of the learning literature and introduce heterogeneity in the reduced-form models rather than in the micro-foundations (see, inter alia, Bullard \& Mitra (2002), Arifovic et al. (2013), Hommes \& Lustenhouwer (2019)). We are well aware of the conceptual limitation of this approach. Nonetheless, while the complications of the alternative are clear (see e.g. Woodford (2013)), the benefits in terms of qualitative results remain uncertain. For instance, in an asset-pricing model, Adam \& Marcet (2011) show that under a sophisticated form of adaptive learning, the infinite-horizon pricing equation reduces to a myopic mean-variance equation. Bearing in mind those caveats, we proceed within the reduced-form model.
} 
where $\phi^{\pi}$ and $\phi^{y}$ are, respectively, the reaction coefficients to the inflation and the output gaps, and $\bar{r} \equiv \pi^{T}+\rho$ the steady-state level of interest rate associated with the inflation target $\pi^{T}$ and the households' discount rate $\rho \equiv-\log (\beta)$.

We now solve the model under the benchmark of RE and then detail how we introduce SL in the expectation formation process of the agents.

\subsection{The model under rational expectations}

In this section, we consider $\mathrm{RE}$ and impose $\mathbb{E}_{j, t}^{*}(\cdot)=E\left(\cdot \mid I_{t}\right)$ to be the rational expectation operator given the information set $I_{t}$ common to all agents in period $t$. We solve for the Minimal State Variable (MSV) solution using the method of undetermined coefficients.

It is well known that the ELB introduces a non-linearity in the Taylor rule and generates an additional deflationary steady-state next to the target (Benhabib et al. 2001). This ELB steady-state corresponds to a liquidity trap where the deflation rate matches the discount factor. Hence, expressing the model in reduced form is challenged by this non-linearity, and we need to disentangle two pieces, one around the target and one when the ELB is binding. ${ }^{9}$

A short digression through the one-dimensional Fisherian model easily illustrates this configuration. Figure 3 displays inflation and interest rate dynamics, abstracting from the production side: the social optimum or inflation target corresponds to $\widehat{\pi}=0$ and the deflationary steady-state to $\widehat{\pi}^{e l b}$. Provided that $\widehat{\pi}^{e l b} \leq 0 \leq \pi^{T}$, the two equilibria co-exist.

\footnotetext{
${ }^{9}$ We follow here the related NK literature and impose the ELB constraint in the log-linearized model around the targeted steady-state to describe the dynamics around the low inflation state, see, inter alia, Nakov (2008), Guerrieri \& Iacoviello (2015). This method gives a second-best estimate of the dynamics around the deflationary steady-state. A first-best would be to log-linearize the model around this second steady-state but would result in an MSV solution involving extra additional state variables (Ascari \& Sbordone 2014) and, hence, additional coefficients to learn under SL (see Section 2.3). However, the benefits in terms of qualitative results are unlikely to outweigh the costs of such a complication of the learning process of the agents.
} 


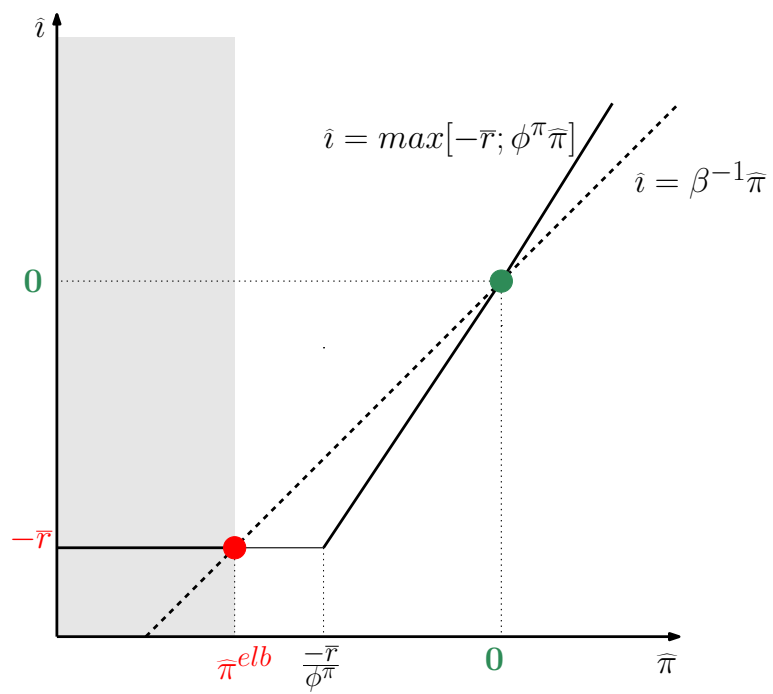

Notes: We can write the log-approximated Fisher equation as follows: $\widehat{\imath}=\beta^{-1} \widehat{\pi}$. At the targeted steady-state (in green), no deviation occurs: $\widehat{\imath}=\beta^{-1} \widehat{\pi}=0$. At the ELB (in red), we can derive an equilibrium such that $-\bar{r}=\beta^{-1} \widehat{\pi}^{\text {elb }} \Leftrightarrow$ $\widehat{\pi}^{e l b}=-\bar{r} \beta$. Provide that $\widehat{\pi}^{e l b} \leq \pi^{T}$, the two equilibria co-exist. The shaded area is indeterminate under RE and unstable under adaptive learning (Evans et al. 2008).

Figure 3: Co-existence of two steady states under the ELB constraint

Coming back to the two-dimensional model, Appendix A shows that the functional form of the Minimal State Variable-Rational Expectation Equilibrium (MSV-REE) at the target reads as:

$$
z_{t}=\left(\widehat{y}_{t} \widehat{\pi}_{t}\right)^{\prime}=a+c \widehat{g}_{t}+d \widehat{u}_{t}
$$

The expression of the matrices $a, c$ and $d$ depends on the steady-state considered.

In the rest of the paper, we consider white noise shocks, i.e. $\rho^{g}=\rho^{u}=0$, so the disturbances $u$ and $g$ are $i . i . d$. processes. We assume that $u$ and $g$ are not observable. In this case, the REE solution (6) boils down to a noisy constant $a$ without endogenous persistence. The presence of a floor on the nominal rate makes the equilibrium law of motion of the economy piece-wise:

$$
z_{t}=\left[\widehat{y}_{t} \widehat{\pi}_{t}\right]^{\prime}=\left\{\begin{array}{l}
a^{T}+\chi^{g} \widehat{g}_{t}+\chi^{u} \widehat{u}_{t}, \text { if } i_{t}>0 \\
a^{e l b}+\chi^{g} \widehat{g}_{t}+\chi^{u} \widehat{u}_{t}, \text { if } i_{t}=0,
\end{array}\right.
$$

where the first case is the law of motion when the ELB is not binding (denoted by 
a ' $\mathrm{T}$ ' superscript), the second case is when the ELB is binding (denoted by a 'elb' superscript), $a^{T}=\left(I-B^{T}\right)^{-1} \alpha^{T}, a^{e l b}=\left(I-B^{e l b}\right)^{-1} \alpha^{e l b}$. In the latter expressions, $I$ is the 2-by-2 identity matrix while $\alpha, B, \chi^{g}$ and $\chi^{u}$ are matrices from the solution of the rational expectation model. The exact expression of these matrices can be found in Appendix A. Note that as variables are normalized with respect to their steady-state values at the target, we have $a^{T}=\left(\begin{array}{ll}0 & 0\end{array}\right)^{\prime}$. We now introduce the expectation formation mechanism under SL.

\subsection{Expectations under social learning}

Under SL, we relax the assumption of homogeneous agents endowed with RE and consider instead a population $J$ of $N$ heterogeneous and interacting agents, indexed by $j=1, \cdots, N$. We now define $\mathbb{E}_{j, t}^{*}(\cdot)=E_{j}^{S L}\left(\cdot \mid I_{j, t}\right)$ to be the expectation operator under SL given the information set $I_{j, t}$ available in period $t$ to agent $j$. The information set is agent specific as it contains, besides the history of past inflation and output gaps up until period $t-1$, the current and past individual forecasts that need not be shared with the whole population. Figure 4 summarizes the intra-period dynamics under SL. We now detail each step.

Individual forecasting rules Following Arifovic et al. (2013, 2018), we assume that agents are endowed with a forecasting rule that involves the same variables as the MSV solution. The form of the rule is the same across agents, but with agent-specific coefficients that they revise over time. In any period $t$, each agent $j$ is therefore entirely described by a two-component strategy $\left[a_{j, t}^{y}, a_{j, t}^{\pi}\right]^{\prime}$ and her expectations read as:

$$
E_{j, t}^{S L}\left\{\hat{z}_{j, t+1}\right\}=\left[\begin{array}{c}
E_{j, t}^{S L}\left\{\hat{y}_{t+1}\right\} \\
E_{j, t}^{S L}\left\{\hat{\pi}_{t+1}\right\}
\end{array}\right]=\left[\begin{array}{c}
a_{j, t}^{y} \\
a_{j, t}^{\pi}
\end{array}\right]
$$




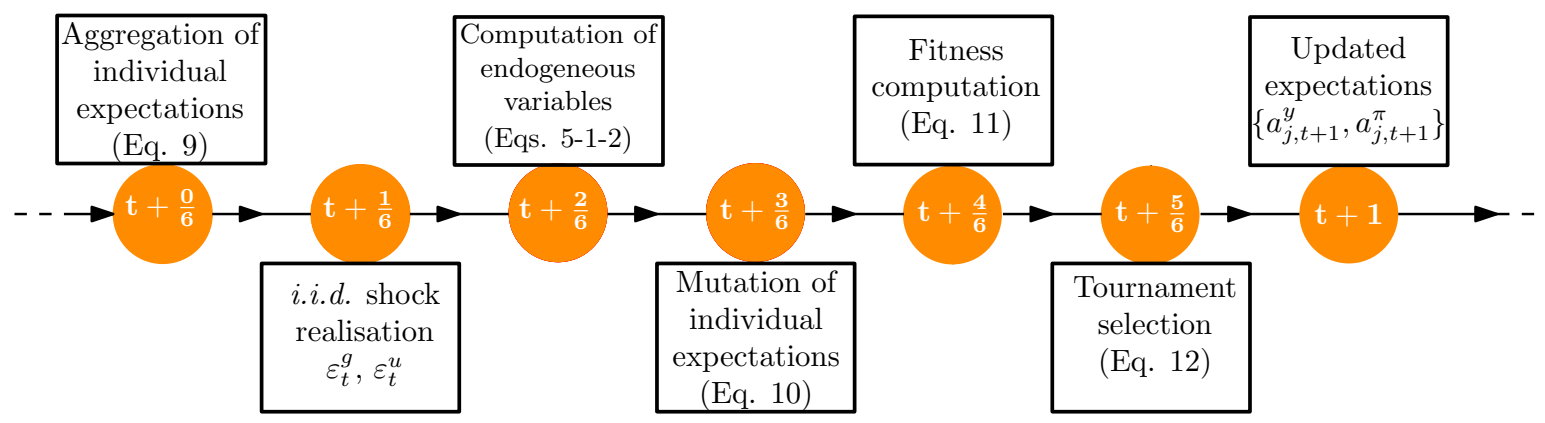

Figure 4: Intra-period timing of events in the model under SL

Those pairs of forecast values find an appealing interpretation. In the absence of shocks, $a_{j}^{y}$ corresponds to her long-run output gap forecast and $a_{j}^{\pi}$ to her long-run inflation gap forecast. In the presence of $i . i . d$. shocks, those values correspond to her average output gap and inflation gap forecasts. Under either of those interpretations, the forecasts $\left[\begin{array}{lll}a_{j, t}^{y} & a_{j, t}^{\pi}\end{array}\right]^{\prime}$ of the agents represent their beliefs about the steady-state values of the inflation and output gaps, which allows us to intuitively measure expectation anchorage or unanchorage by simply evaluating the distance between those forecasts values, and their targeted counterparts (i.e. zero). ${ }^{10}$ On empirical grounds, heterogenous coefficients $\left[a_{j, t}^{y} a_{j, t}^{\pi}\right]$ may also capture the disagreement among forecasters observed in survey data. In particular, dispersed coefficients on inflation $a_{j, t}^{\pi}$ can be interpreted as disagreement about the CB target, as the latter typically coincides with the inflation steady-state in the workhorse NK model. This disagreement has been empirically documented by surveys conducted by Coibion et al. (2018) for both firms and households in the US.

\footnotetext{
${ }^{10}$ In the sequel, we denote by $\Omega$ such an indicator of expectation anchorage. Specifically, we use the average squared distance of individual expectations to zero: $\Omega_{t}^{\mathbb{E} \pi}=\frac{1}{N} \sum_{j=1}^{N} \mathbb{E}_{j, t}^{S L}\left\{\widehat{\pi}_{t+1}\right\}^{2}$ and $\Omega_{t}^{\mathbb{E} y}=\frac{1}{N} \sum_{j=1}^{N} \mathbb{E}_{j, t}^{S L}\left\{\widehat{y}_{t+1}\right\}^{2}$. The lower those values, the stronger the anchorage of expectations.
} 
Aggregation of individual forecasts Following further Arifovic et al. (2013, 2018), individual expectations (8) are aggregated using the arithmetic mean as:

$$
E_{t}^{S L} \widehat{z}_{t+1}=\frac{1}{N} \sum_{j=1}^{N} \mathbb{E}_{j, t}^{S L} \widehat{z}_{t+1}
$$

and inserted into the reduced-form model (17) to obtain the dynamics of the endogenous variables under SL. Under this aggregation procedure, agents have the same relative weight in expectations formation, thus one agent cannot influence market expectations if the number of agents $N$ is large enough. To have a sizable effects on market expectations, a sentiment or news must spread to a large enough fraction of the population to generate expectation-driven fluctuations. We now detail how agents interact and how their forecasts evolve as sentiments to diffuse in the population.

Agents collectively explore the space of possible forecast values $\left[\begin{array}{ll}a^{y} & a^{\pi}\end{array}\right]^{\prime}$. Specifically, this class of learning models utilizes two operators.

Mutation The first one is a stochastic innovation process, or mutation, that allows for a constant exploration of the state space outside the existing population of forecasts. In each period, each agent's forecasts are modified by an idiosyncratic shock with exogenously given probabilities. Her output gap forecast is modified with probability $\mu_{y}$ and her inflation gap forecast with probability $\mu_{\pi}$. In short, her forecasts of any variable $x=\{y, \pi\}$ modified in any period as follows:

$$
a_{j, t+1}^{x}=\left\{\begin{array}{l}
a_{j, t}^{x}+\iota_{j, t} \xi^{x} \text { with probability } \mu_{x} \\
a_{j, t}^{x} \text { with probability } 1-\mu_{x}
\end{array}\right.
$$

with $\iota_{j, t}$ an idiosyncratic random draw from a standard normal distribution with standard deviation $\xi^{x}$. In other words, mutations occur in the neighborhood of the individual strategies, and the size of this neighborhood is directly tuned by parameter $\xi^{y}$ along the output dimension and $\xi^{\pi}$ along the inflation dimension. The larger those 
parameters, the wider the neighborhood to be explored around the existing strategies $a_{j, t}^{y}$ and $a_{j, t}^{\pi}$. Mutation can be interpreted as an innovation, a trial-and-error process or a control error in the computation of the corresponding expectations.

Tournament and computation of forecasting performances The second operator, the tournament, is the selection force of the learning process and allows betterperforming strategies to spread among the population at the expense of lower-performing ones. Performance of any forecasting rule $\left[a_{j, t}^{y}, a_{j, t}^{\pi}\right]^{\prime}$ is evaluated using the forecast errors over the whole past history of the economy (not solely over the last period) given the stochastic nature of the environment (see Branch \& Evans 2007).

For each agent $j$, her forecast $a_{j, t}^{x}$ of each variable $x=\{y, \pi\}$ is assessed regarding forecast errors. To each strategy $a_{j, t}^{x}$ is assigned a so-called fitness, computed as:

$$
F_{j, t}^{x}=-\sum_{\tau=0}^{t}\left(\rho^{x}\right)^{\tau}\left(\widehat{x}_{t-\tau}-a_{j, t}^{x}\right)^{2}
$$

The terms $\widehat{y}_{t-\tau}-a_{j, t}^{y}$ and $\widehat{\pi}_{t-\tau}-a_{j, t}^{\pi}$ correspond, respectively, to the output and inflation gap forecast errors that agent $j$ would have made in period $t-\tau-1$, had she used her current forecast values $a_{j, t}^{y}$ and $a_{j, t}^{\pi}$ to predict the output and inflation gaps in period $t-\tau$. The smaller the forecast errors, the higher the fitness.

Parameter $\rho^{x} \in[0,1]$ (for $x=y, \pi$ ) represents memory. In the nested case where $\rho^{x}=0$, the fitness of each strategy is completely determined by the forecast error on the most recent observable data, i.e. $t$ given our timing of events (see, again, Fig. 4). For any $0<\rho^{x} \leq 1$, all past forecast errors impact the fitness but with exponentially declining weights while, for $\rho^{x}=1$, all past errors have an equal weight in the computation of the fitness. This memory parameter allows the agents to discriminate between a one-time lucky draw and persistently good forecasting performances.

In the tournament, agents are randomly paired (the number of agents is conveniently chosen even), their fitness on inflation and output gap forecasts are each compared and 
the one with the lowest fitness copies the forecast of the other. There are two separate tournaments: one for inflation gap forecasts $\left\{a_{j, t}^{\pi}\right\}_{j \in J}$ and one for output gap forecasts $\left\{a_{j, t}^{y}\right\}_{j \in J}$. Formally, for each pair of agents $k, l \in J, k \neq l$, with individual forecasts $a_{k, t}^{x}$ and $a_{l, t}^{x}(x \in\{\pi, y\})$, the tournament operates an imitation of the more successful forecasts as follows:

$$
\begin{aligned}
& a_{k, t+1}^{x}=a_{l, t+1}^{x}=a_{k, t}^{x} \text { if } F_{k, t}^{x}>F_{l, t}^{x} \\
& a_{k, t+1}^{x}=a_{l, t+1}^{x}=a_{l, t}^{x} \text { if } F_{k, t}^{x} \leq F_{l, t}^{x}
\end{aligned} \text { for } x \in\{\pi, y\} .
$$

The tournament occurs after the mutation operator in order to screen out badperforming forecasts stemming from mutation. This allows the model to be less sensitive to the parameter values tuning mutation (i.e. the probabilities $\mu_{x}$ and the size $\xi^{x}$, $x=\{y, \pi\})$ than if mutation were to take place after the tournament selection, and all newly created forecasts were to determine aggregate expectations without consideration of their performances. This way, the mutation process can be more frequent and of wider amplitude so as to allow for a faster adaptation of the agents to new macroeconomic conditions, while limiting the amount of noise introduced by the SL algorithm.

Simulation protocol We study the dynamics of the model using numerical simulations. Throughout the rest of the paper, we proceed as described in Arifovic et al. (2013, 2018). We generate a history of 100 periods along the law of motion of the economy around the target (see Eq. (7)) and introduce a population of SL agents in $t=100$. Their initial forecasts are drawn from the same support as the one used in the mutation process, i.e. from a normal distribution with standard deviation $\xi^{x}, x=\pi, y$. The first 100 periods are used to provide the agents with a history of past inflation and output gaps in order to compute the fitness of their newly introduced forecasts. In the simulations exercises in the next sections, we vary the initial average of the normal distribution to tune the degree of pessimism in the economy. The further below zero the 
initial average forecasts are, the more pessimistic views the agents hold about future inflation and output gaps.

Finally, it is important to recognize that the RE representative agent benchmark is nested in our heterogeneous-agent model: as soon as the inflation and output gap expectations of all agents are initialized at the targeted values and mutation is switched off (i.e. $\left.\xi^{y}, \xi^{\pi}=0\right)$, the dynamics boil down to the RE benchmark. Under SL, our model involves a few parameters, namely the probabilities of mutation, the sizes of those mutations and the memory of the fitness function. We now detail how we estimate those parameter values.

\section{$3 \quad$ Estimation of the model under social learning}

We jointly estimate the learning parameters and the structural parameters of the model. We first describe our choice and construction of the dataset, then discuss our estimation method and, finally, present the results.

\subsection{Dataset}

Macroeconomic US time series for output, price index and nominal rates are taken from the FRED database. Forecast data come from the SPF of the Federal Reserve of Philadelphia. This choice is usual in the related literature, as it is argued that those data provide a good approximation of the private sector expectations that are implicitly involved in the New Keynesian micro-foundations (Del Negro \& Eusepi 2011). SPF data span the period from 1968 to 2018 on a quarterly basis. To make the dataset stationary, we divide output by both the working age population and the price index. In order to obtain a measurement of the output gap, we compute the percentage deviations of the resulting output time series from its linear trend. The inflation rate is measured by the growth rate of the GDP deflator. 
As heterogeneity in expectations drives the dynamics of the SL model, we construct an empirical measure of that heterogeneity in the survey data. We use the crosssectional dispersion of the individual forecasts in order to obtain time series of forecasts' heterogeneity and compute the standard deviation of the individual forecasts among all participants in each period of the survey.

\subsection{Estimation method}

With those data at hand, we proceed by matching the statistics from empirical moments with their simulated counterparts under SL. We provide technical details of our estimation method in Appendix C. In short, we use the Simulated Moments Method (SMM) as initially developed by McFadden (1989), which provides a rigorous basis for evaluating whether the model is able to replicate salient business cycle properties. $^{11}$

In order to avoid identification issues, the number of estimated parameters has to be equal to the number of matched moments so that each estimated parameter can be directly mapped onto one empirical moment. Hence, we first have to reduce the number of dimensions of the matching problem and calibrate some of the parameters, namely the monetary policy and the preference parameters, as is standard in the related macroeconomic literature, and the number of agents (see Table 1).

We are left with four structural parameters from the NK model, namely the size of the fundamental shocks $\sigma^{g}$ and $\sigma^{u}$, the slope of the NK Phillips curve (parameter $\kappa$ ) and the natural rate $\bar{r}$. As we have calibrated the value of the discount factor $\beta$ (see Table 1), we estimate the value of the inflation trend over the period considered, which uniquely determines the value of $\bar{r} .{ }^{12}$ As for the SL parameters, we need not estimate

\footnotetext{
${ }^{11}$ Due to the non-linearity introduced by the ELB, we may not apply the Kalman filter and would need to use a non-linear filter to estimate the model with Bayesian full-information techniques. Given that this paper is the first attempt to bring such a heterogeneous-expectation model to the data, we encountered additional difficulties in estimating the SL model with an SMM (see Appendix C). In particular, the SL algorithm brings an additional non-linearity into the piecewise-linear model and an additional source of stochasticity next to the fundamental shocks. Hence, we have left the perspective of Bayesian estimation for future research.

${ }^{12}$ Strictly speaking, $\bar{r}$ is associated with the inflation target per Eq. (5) but no such target existed
} 


\begin{tabular}{|c|c|c|c|}
\hline & & Values & Sources \\
\hline$\sigma$ & intertemporal elasticity of substitution & 1 & Galí (2015) \\
\hline$\phi^{\pi}$ & policy stance on inflation gap & 1.50 & Galí (2015) \\
\hline$\phi^{y}$ & policy stance on output gap & 0.125 & Galí (2015) \\
\hline$\beta$ & discount factor & 0.995 & Jarociński \& Maćkowiak (2018) \\
\hline$N$ & number of agents & 300 & Arifovic et al. (2018) \\
\hline
\end{tabular}

Table 1: Calibrated parameters (quarterly basis)

common values for the inflation and the output gap expectation processes as the two tournaments are separated and the two time series are likely to behave differently and exhibit different properties, both in reality and in the model. For instance, estimating inflation and output gap-specific memory parameters $\rho^{\pi}$ and $\rho^{y}$ may translate the fact that agents can learn that one variable may display more persistence than the other. Hence, we estimate six learning parameters, namely the mutation sizes and frequencies $\xi^{x}$ and $\mu_{x}$ as well as the memory of the fitness measures $\rho^{x}$ for $x=\{\pi, y\}$.

We now discuss the mapping between those parameters and the empirical moments to match. First, the standard deviations of the shocks $\sigma^{g}$ and $\sigma^{u}$ naturally capture the empirical volatility of output and inflation. Second, the inflation trend $\bar{\pi}$ aims to match the ELB probability. To see why, recall that a higher natural rate $\bar{r}$ mechanically decreases the probability of hitting the ELB, as the latter is defined as $\widehat{\imath_{t}}=-\bar{r}$, which is strictly decreasing in the value of the inflation target. Finally, the slope of the Phillips curve $\kappa$ determines the correlation between the output and inflation gaps per Eq. (2).

As for the SL parameters, the memories of the fitness function $\rho^{y}$ and $\rho^{\pi}$ tune the sluggishness of the expectations because they determine the weights on recent versus past forecast errors in the computation of the forecasting performances. The higher $\rho^{y}$ and $\rho^{\pi}$, the longer the memory of the agents, the less reactive the learning process to recent errors and the more sluggish the expectations. As sluggishness in expectations is the only source of persistence in the model once we consider i.i.d. shocks, parameters in the US for most of the time period considered. Therefore, we estimate an inflation trend over that period. 
$\rho^{y}$ and $\rho^{\pi}$ are matched with the autocorrelation of, respectively, the output and the inflation gaps.

The remaining four learning parameters control the mutation processes that are the source of the pervasive heterogeneity in expectations in the SL model. We understandably use those parameters to match four moments characterizing heterogeneity in the SPF data: the average dispersion of the output and the inflation gap forecasts over the time period considered, denoted respectively by $\Delta^{\mathbb{E} y}$ and $\Delta^{\mathbb{E} \pi}$, and their first-order autocorrelations, denoted by $\rho\left(\Delta_{t}^{\mathbb{E} y}, \Delta_{t-1}^{\mathbb{E} y}\right)$ and $\rho\left(\Delta_{t}^{\mathbb{E} \pi}, \Delta_{t-1}^{\mathbb{E} \pi}\right)$. In line with intuition, sensitivity analyzes of the objective function of the matching problem with respect to those learning parameters have reported the following associations: the mutation sizes $\xi^{y}$ and $\xi^{\pi}$ capture a substantial share of the empirical dispersion of output and inflation gap forecasts, while the mutation frequencies $\mu^{y}$ and $\mu^{\pi}$ match most of their autocorrelation.

Finally, in the same vein as Ruge Murcia (2007), we impose prior restrictions on the estimated parameters and treat them as additional moments in the objective function. The details are deferred to Appendix C. The priors for the structural NK parameters are taken from the literature on Bayesian estimation of DSGE models (Smets \& Wouters 2007) and we choose priors for the learning parameters that are in line with the values used in the SL literature such as Arifovic et al. (2013) (see Table 3).

\subsection{Estimation results}

Table 2 reports the matched moments and their empirical counterparts (in percentage points). Table 3 gives the corresponding estimated values of the parameters.

It is first striking to see how the simple two-dimensional model delivers remarkably good matching performances along the ten stylized facts considered. The estimated model accounts for a substantial share of all ten moments. For half of them, the simulated moments even fall within the confidence interval of their empirical counterparts, 
which means that our model replicates those moments fully. We succeed in capturing not all, but a non-negligible part, of the persistence in macroeconomic variables with a model that employs only white-noise shocks. ${ }^{13}$ We shed further light on the source of that persistence in Section 4.1, but at that stage, we can state that learning acts as an endogenous propagation mechanism that amplifies the effects of i.i.d. shocks and accounts for $22 \%$ of the empirical output gap persistence and even $63 \%$ of the inflation persistence found in the data.

Furthermore, all simulated correlations are of the same sign as their observed counterparts. Our model succeeds in producing positive autocorrelation in forecast dispersion. This result is an important step forward in the modeling and estimation literature as we show that our simple framework can address the empirical heterogeneity in expectations that is not part of the RE material.

The model also matches particularly well the probability of the ELB on nominal interest rates to bind despite the relatively modest amplitude and i.i.d. structure of the fundamental shocks. Those ELB episodes are not the result of large exogenous shocks but are an endogenous product of the interplay between learning and those small i.i.d. shocks. For illustrative purposes, Figure 5 displays the time series of the endogenous variables and the expectations from a representative simulation of the model. We can see an occasionally binding ELB around periods 30 to 60 that coincides with belowtarget expectations. Before detailing in the next section how such dynamics can arise from the amplification mechanism induced by SL, we briefly discuss the estimated values of the parameters in Table 3.

All our estimated values are consistent with empirical values and usual estimates. For instance, the estimated value of the slope of the Phillips curve $(\kappa)$ is in line with

\footnotetext{
${ }^{13}$ Matching all the persistence would not be a realistic or desirable objective: it is unlikely that all macroeconomic persistence stems from learning in expectations and our model ignores all other fundamental sources of persistence in the economy. We rather provide a measure of the share of the persistence that can be attributed to learning.
} 


\begin{tabular}{|c|c|c|c|c|}
\hline \multirow{2}{*}{\multicolumn{2}{|c|}{ Matched moments }} & \multicolumn{2}{|c|}{ Moments } & \multirow{3}{*}{$\begin{array}{c}\text { Empirical } \\
\text { Conf.int. }\end{array}$} \\
\hline & & Empirical $\mathcal{M}_{O}$ & Simulated $\mathcal{M}_{S}$ & \\
\hline$\overline{\sigma\left(\hat{y}_{t}\right)}$ & - output gap sd. & 4.38 & 4.39 & \\
\hline$\rho\left(\hat{y}_{t}, \hat{y}_{t-1}\right)$ & - output gap autocorr. & 0.98 & 0.22 & {$[0.98-0.99]$} \\
\hline$\sigma\left(\hat{\pi}_{t}\right)$ & - inflation gap sd. & 0.6 & 0.66 & {$[0.54-0.66]$} \\
\hline$\rho\left(\hat{\pi}_{t}, \hat{\pi}_{t-1}\right)$ & - inflation gap autocorr. & 0.9 & 0.56 & {$[0.87-0.92]$} \\
\hline$\rho\left(\hat{\pi}_{t}, \hat{y}_{t}\right)$ & - inflation-output correlation & 0.08 & 0.097 & {$[-0.07-0.21]$} \\
\hline$\Delta^{\mathbb{E} y}$ & - av. forecast dispersion of output gap & 0.36 & 0.4 & {$[0.31-0.41]$} \\
\hline$\Delta^{\mathbb{E} \pi}$ & - av. forecast dispersion of inflation gap & 0.25 & 0.20 & {$[0.22-0.28]$} \\
\hline$\rho\left(\Delta_{t}^{\mathbb{E} y}, \Delta_{t-1}^{\mathbb{E} y}\right)$ & - autocorr. of forecast disp. of output gap & 0.76 & 0.63 & {$[0.70-0.82]$} \\
\hline$\rho\left(\Delta_{t}^{\mathbb{E} \pi}, \Delta_{t-1}^{\mathbb{E} \pi}\right)$ & - autocorr. of forecast disp. of inflation gap & 0.64 & 0.4 & {$[0.55-0.72]$} \\
\hline$P\left(i_{t}>0\right)$ & - probability not at the ELB & 0.86 & 0.83 & {$[0.81-0.91]$} \\
\hline \multicolumn{2}{|c|}{ Objective function } & $\times$ & 0.85 & $\times$ \\
\hline
\end{tabular}

Notes: The values in brackets are the confidence interval at $99 \%$ of the empirical moments.

Table 2: Comparison of the (matched) theoretical moments with their observable counterparts

\begin{tabular}{|c|c|c|c|c|c|c|}
\hline \multirow{2}{*}{\multicolumn{2}{|c|}{ Estimated Parameters }} & \multicolumn{3}{|c|}{ Prior Distributions } & \multicolumn{2}{|c|}{ Posterior Results } \\
\hline & & Shape & Mean & STD & Mean & STD \\
\hline$\sigma^{g}$ & - real shock std & Invgamma & .1 & 5 & 3.8551 & $5.1 \mathrm{e}-06$ \\
\hline$\sigma^{u}$ & - cost-push shock std & Invgamma & .1 & 5 & 0.4232 & $4.1 \mathrm{e}-06$ \\
\hline $\bar{\pi}$ & - quarterly inflation trend & Beta & .62 & .1 & 0.829 & $7.7 \mathrm{e}-06$ \\
\hline$\kappa$ & - Phillips curve slope & Beta & .05 & .1 & 0.0095 & $4 \mathrm{e}-06$ \\
\hline$\mu^{y}$ & - mutation rate for $\mathbb{E} y$ & Beta & .25 & .1 & 0.2467 & $4.6 \mathrm{e}-06$ \\
\hline$\mu^{\pi}$ & - mutation rate for $\mathbb{E} \pi$ & Beta & .25 & .1 & 0.2748 & $6 e-06$ \\
\hline$\xi^{y}$ & - mutation std. for $\mathbb{E} y$ & Invgamma & .1 & 2 & 0.8547 & $3.3 \mathrm{e}-06$ \\
\hline$\xi^{\pi}$ & - mutation std. for $\mathbb{E} \pi$ & Invgamma & .1 & 2 & 0.7406 & $1.9 \mathrm{e}-06$ \\
\hline$\rho^{y}$ & - fitness decay rate for $\mathbb{E} y$ & Beta & .5 & .2 & 0.8301 & $9.4 \mathrm{e}-06$ \\
\hline$\rho^{\pi}$ & - fitness decay rate for $\mathbb{E} \pi$ & Beta & .5 & .2 & 0.5465 & $5.4 \mathrm{e}-06$ \\
\hline
\end{tabular}

Notes: The low values of the standard deviation of the estimated parameter values only indicate that the algorithm has converged; they do not translate into confidence intervals.

Table 3: Estimated parameters using the simulated moment method matching the SPF data $(1968-2018)$

Woodford (2003) and is also consistent with the structural flattening underlying the most recent measures (Gourio et al. 2018). The estimated (yearly) inflation trend is $3.4 \%$, which nicely falls into the range between the average inflation rate over the time span considered that includes the 1970s (4.3\%) and the Fed inflation target that was adopted later $(2 \%)$. Next, given the calibrated discount factor $\beta$, the implied value for the (yearly) natural interest rate is $5.45 \%$, which is close to the average federal funds rate over the sample (namely $5.2 \%$ ).

As for the estimated values of the mutation parameters of SL, we can see that 
a. Inflation gap $\hat{\pi}_{t}$

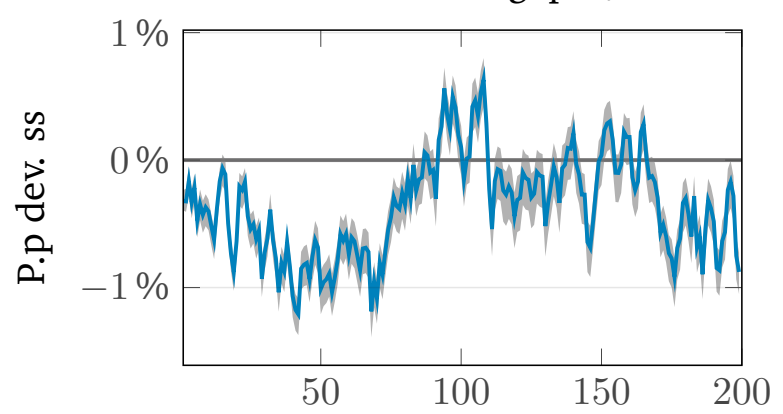

c. Mean inflation expectations $E_{t}^{S L} \hat{\pi}_{t+1}$ b. Output gap $\hat{y}_{t}$

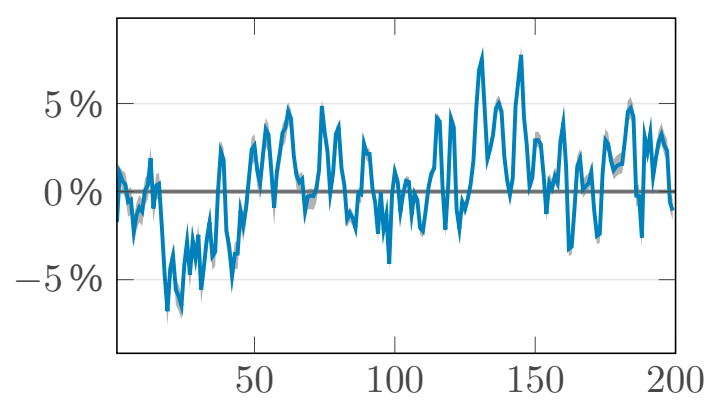

d. Mean output expectations $E_{t}^{S L} \hat{y}_{t+1}$
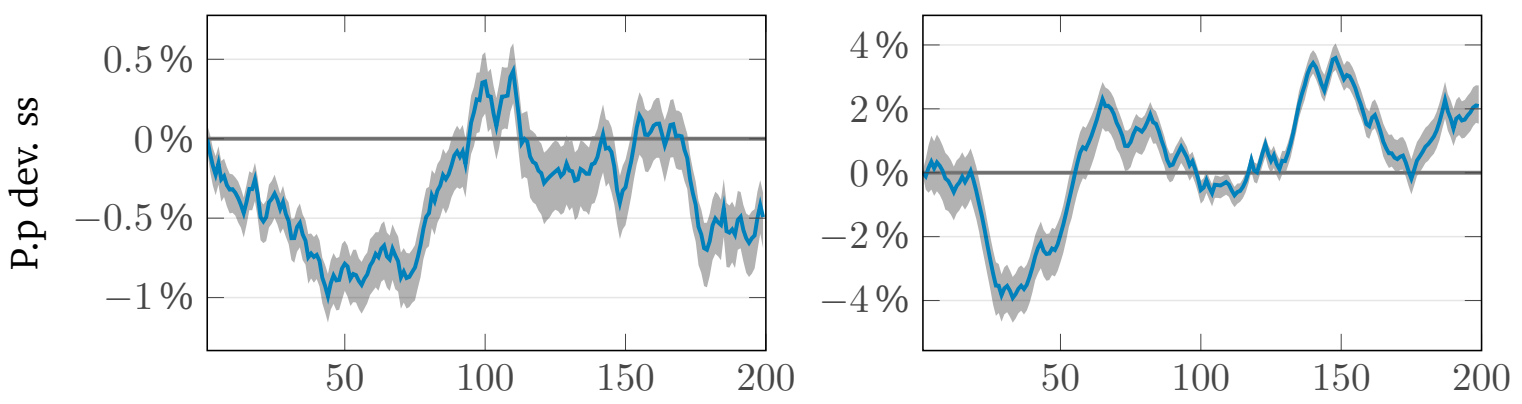

e. Dispersion inflation expectations $\Delta_{t}^{\pi}$

f. Dispersion output expectations $\Delta_{t}^{y}$
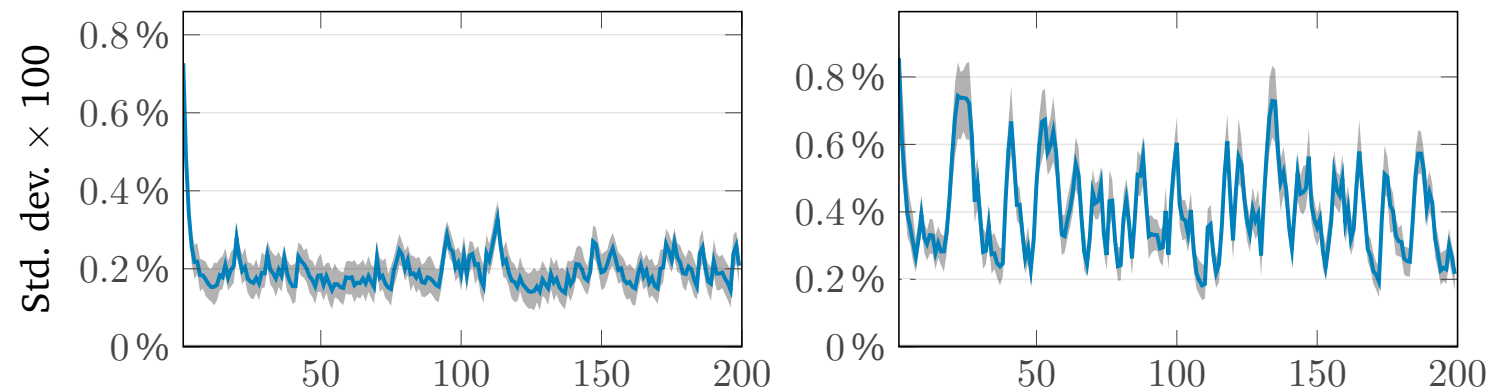

g. Nominal interest rate $\hat{i}_{t}$
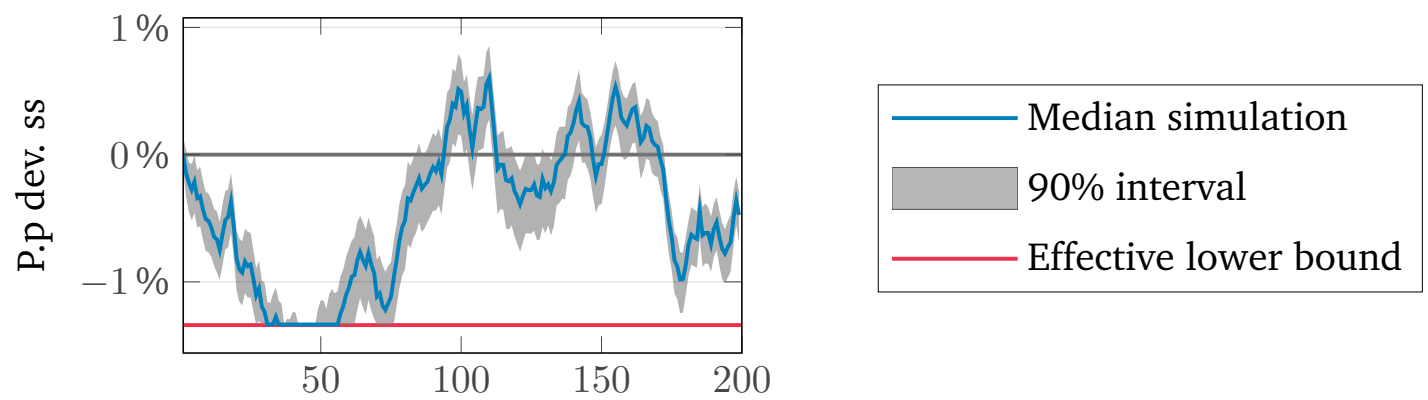

Notes: In the direction of reading, time series of the inflation gap, the output gap, the average inflation gap expectations, the average output gap expectations, the cross-sectional dispersion of output gap expectations, the cross-sectional dispersion of inflation gap expectations and the nominal interest rate. The blue line represents the median realizations over 1,000 runs and the shaded areas represent the 5th and the 95th percentiles.

Figure 5: Representative time series from a simulation of the model under SL 
they are all in line with the values usually employed in numerical simulations in the related literature (Arifovic et al. 2013). The estimated values of $\rho^{y}$ and $\rho^{\pi}$ imply that agents' memory is bounded, ${ }^{14}$ which is highlighted by experimental evidence (Anufriev \& Hommes 2012) and empirical estimates from micro data (Malmendier \& Nagel 2016).

We conclude with our first major contribution: our parsimonious model is able to jointly and accurately reproduce ten salient features of macroeconomic time series and survey data, including the ELB duration and the pervasive heterogeneity in forecasts, while using plausible parameter values. We now proceed to the analysis of the underlying propagation mechanism in the model induced by SL.

\section{Dynamics under social learning}

This section first analyzes the stability properties of the targeted steady-state under SL. To unravel the dynamics of expectations, we then look at a specific transitory path back towards the target after an adverse expectational shock. Next, we systematically compare the business cycles properties under SL and RE and assess the welfare loss entailed by heterogeneous expectations with respect to the RE representative agent benchmark.

\subsection{Stability analysis}

We examine here the asymptotic behavior of the model over the entire state space of the endogenous variables $(\hat{\pi}, \hat{y})$, as utilized in the introduction (see, again, Fig. 2). We proceed through Monte Carlo simulations. Figure 6 represents the phase diagram of the model where the average inflation gap expectation (i.e. the average of the $\left\{a_{j}^{\pi}\right\}$ values across agents) is given on the $\mathrm{x}$-axis and the average output gap expectation (i.e. the

\footnotetext{
${ }^{14}$ If one discards observations weighting less than $1 \%$, we have $0.83^{25}<0.01$ and $0.54^{7}<0.01$, which implies that agents' memory amounts to roughly 25 quarters for forecasting the output gap and 7 quarters for forecasting the inflation gap.
} 
average of the $\left\{a_{j}^{y}\right\}$ values) on the y-axis. The initial strategies are drawn around each point of the state space, and we repeat each initialization configuration 1,000 times with different seeds of the Random Number Generator (RNG). We obtain the phase diagram by imposing a one-time expectational shock from the target to each point of the state space and then assess whether inflation and output gaps converge back on the targeted steady-state (see Fig. 6a) and if so, at which speed (see Fig. 6b). The two figures show that the model either converges to the target (in gray-shaded areas) or diverges along a deflationary spiral (in white areas).

The main message from that exercise is that the basin of attraction of the target under SL is larger than the determinacy region of the targeted steady state under RE. To see that, notice that there is a considerable locus of points on the left-hand side of the stable manifold associated with the saddle point under recursive learning (red dashed line in Fig. 6) from where the model converges back to the target under SL. By contrast, we know from the related literature that this manifold marks the frontier between (local) determinacy and indeterminacy under RE. It also marks the frontier between (local) E-stability and divergence under adaptive learning (see Evans et al. (2008) and Appendix B for further details and references).

A wider stability region of the target under SL than under recursive learning is due to a key difference between the two expectation formation mechanisms (see also the related discussions in Arifovic et al. $(2013,2018))$. An adaptive learning algorithm is not concerned with alternative forecasting solutions. By contrast, under SL, expectations are heterogeneous at any point in time: a diversity of forecasts, some more and some less pessimistic than the average of the population, always co-exist and have a chance to spread through the selection pressure of the evolutionary algorithm. Among that diversity, only the forecasts that deliver the lowest forecast errors over the past history (and not just the most recent period) survive and feed back into the dynamics of the endogenous variables. Hence, a single inflation and output gap data point in the 


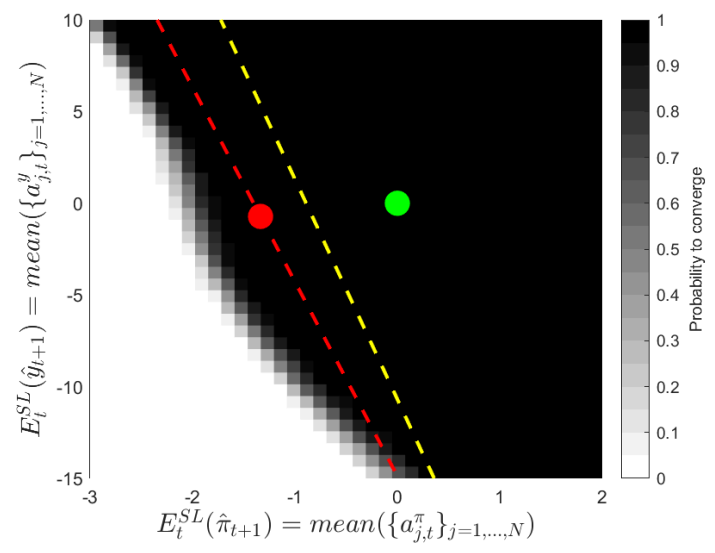

(a) Stability of the target under SL

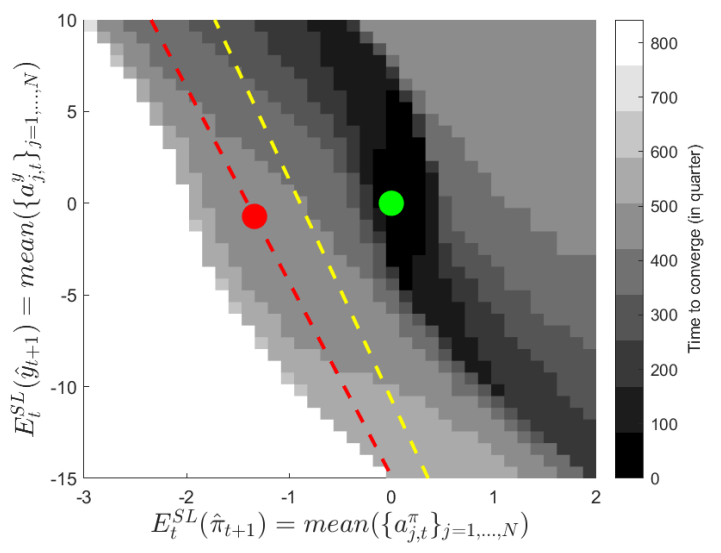

(b) Speed of convergence to the target under SL

Notes: See explanations at the end of Section 2.3. The simulations proceed for 1,000 periods. We perform 1,000 simulations per grid point of the state space. The targeted steady-state is denoted by the green dot, and the deflationary steady-state by the red one. The ELB frontier (yellow dashed line) is the locus of points for which $-\bar{r}=\phi^{\pi} \widehat{\pi}+\phi^{y} \widehat{y}$ : on the left-hand side, the ELB binds. The stable manifold associated with the saddle low inflation steady-state (red line) is computed under recursive learning and corresponds to the stable eigenvector of $B^{e l b}$ : on the left-hand side, the model is indeterminate under $\mathrm{RE}$ and E-unstable. The empty area represents pairs of expectation values for which the model diverges along a deflationary spiral.

Left: The darker, the higher the probability to converge back to the steady-state. Right: The darker, the faster the convergence back to the steady-state.

Figure 6: Global dynamics under social learning

unstable region caused by a one-time pessimistic shift of the average forecasts is not enough to steer the whole population of forecasts beyond the stable manifold, along a deflationary path.

Coming back to Figure 6, even after a strong pessimistic shift, some individual forecasts are below the target but still lie above the stability frontier (again, the red line in Fig. 6), i.e. they are mildly pessimistic, while most individual forecasts lie beyond the frontier, in the indeterminacy region, i.e. they are the most pessimistic. Because the mildly pessimistic forecasts deliver lower forecast errors than the most pessimistic forecasts when it comes to forecasting on average over the whole history, which includes pre-shock dynamics, they spread out and steer the economy back to the target.

Under adaptive learning, a single forecast in the indeterminacy region would result in a negative forecast error, i.e. realized inflation and output gaps decline even further 
below their expected values as they diverge in that region of the state space. This negative forecast error causes agents to revise down their expectations even further, which eventually drives the economy along a deflationary spiral. Yet, our model may also lead to self-sustaining deflationary spirals when shifts in expectations are large enough to throw the entire population of strategies beyond the stable manifold. In such an extreme case, a deflationary trend kicks in. However, for this to happen, as shown by the white area in Figure 6a, the one-time shift in expectations has to be implausibly large.

Another related interesting observation is given by Figure 6b. Using the same state space as Figure 6a, the figure reports the speed of convergence to the target for each pair of initial average expectations. The darker the area, the faster the convergence. It is striking to see that the closer expectations to the targeted steady-state, the faster the convergence. In general, there is a locus of points spiraling around the target where convergence is fast, which is consistent with the complex eigenvalues associated with that steady-state. In contrast, the further the expectations from the target, the slower the convergence.

Most interestingly, the area in the southwest side from the target, beyond the stability frontier, is depicted in light gray. This means that for those severely pessimistic inflation and output gap expectations, the model under SL does converge back to the target, but does so at a particularly slow speed. This area is beyond the ELB frontier (yellow dashed line), which indicates that the ELB is binding yet the model does not diverge along a depressive downward spiral.

Those observations show that our model can produce persistent but non-diverging episodes at the ELB, and heterogeneity in expectations plays an essential role in generating those dynamics. We now analyze in detail the characteristics of a transitory path to the target to show how the interplay between learning and the endogenous variables creates those persistent but stable dynamics. 


\subsection{Response to a pessimistic shock}

To shed more light on the properties of the model under SL, we look at a specific expectational shock and study how it propagates in the model. This shock can be interpreted as a sudden pessimistic change in the sentiment of households and firms (or negative news), which now expect a recession (Schmitt-Grohé \& Uribe 2017). In our stability graph, this boils down to plotting the transitory dynamics from one specific point of Figure 6 back to the target. Specifically, we simulate a one-time pessimistic shock on output gap expectations of all agents that is large enough to shift the average expectations beyond the boundary of the stability frontier depicted in Figure $6 .{ }^{15}$ We can then investigate why and how the system converges back to the target under SL from a point of the state space where it would not under some form of recursive learning.

The main outcome of such a shock is a prolonged depressive episode at the ELB (see Fig. 7): inflation and interest rates exhibit considerable persistence below their target while output gap recovers faster, and even temporarily overshoots the steady state. These dynamics entailed under SL are empirically much closer to the recent economic experience discussed in the introduction than the excess volatility in the indeterminacy region under RE or the diverging deflationary paths under adaptive learning.

Let us now unravel the underlying forces at play under SL that deliver those empirically appealing dynamics. The initial deviations from steady-state are triggered by the pessimistic shock only, while the resulting prolonged low inflation and ELB environment stems entirely from the sluggish dynamics of expectations under SL and their self-fulfilling nature in the NK model.

As explained in Section 4.1, right after the shock, the most pessimistic forecasts are discarded at the profit of mildly pessimistic but below-target forecasts. This elimination of the most negative forecasts rules out the possibility of deflationary spirals and

\footnotetext{
${ }^{15} \mathrm{~A}$ more realistic approach would be to shock expectations on both output and inflation, but for the purpose of clarity, we limit the analysis to a single shock. In our simulation, agents expect on average a negative output gap of $14 \%$ to reach the ELB while keeping inflation forecasts at the target.
} 

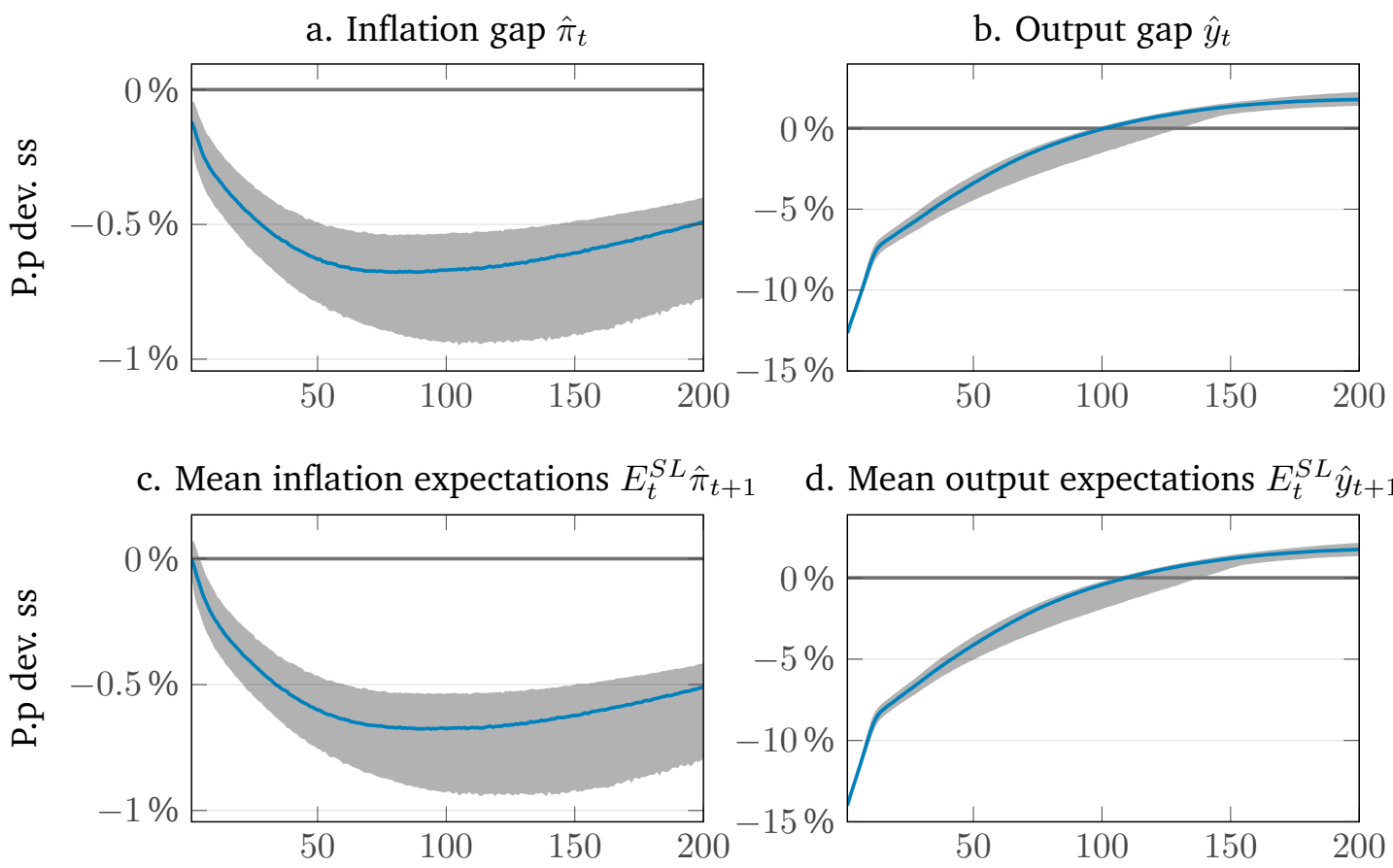

d. Mean output expectations $E_{t}^{S L} \hat{y}_{t+1}$

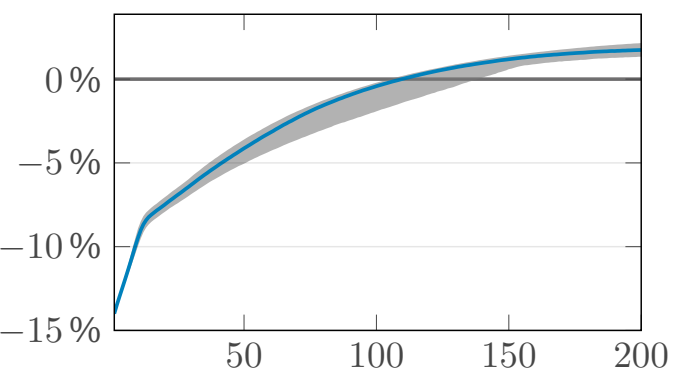

e. Dispersion inflation expectations $\Delta_{t}^{\pi}$

f. Dispersion output expectations $\Delta_{t}^{y}$
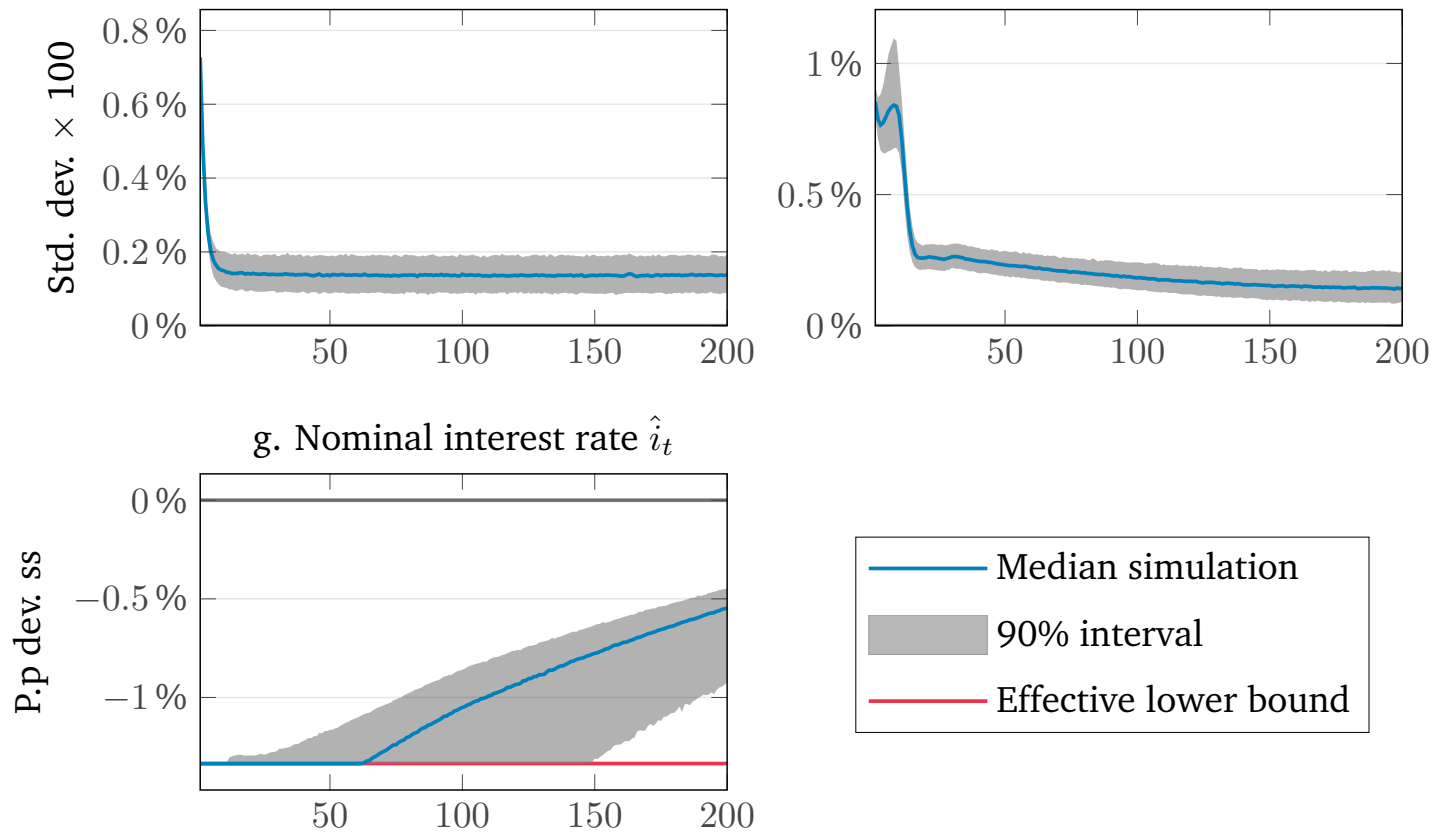

Notes: From the top left to the bottom right, impulse response functions (IRFs) of inflation gap, output gap, average inflation gap expectations, average output gap expectations, nominal interest rate, standard deviation of individual inflation expectations and standard deviation of individual output gap expectations. The blue plain line represents the median realization, and the dotted lines are the $5 \%$ and $95 \%$ confidence intervals over 1,000 Monte Carlo simulations. All plots report the zero line. The lower horizontal line on the IRF of $\hat{\iota_{t}}$ is the ELB.

Figure 7: Impulse response functions (IRFs) of the estimated model to a one-time $-14 \%$ output gap expectation shock 
generates the 'missing disinflation' along the bust. Per their self-fulfilling nature, those mildly pessimistic views nurture the downturn and turn self-confirming, which triggers an accommodating response from the $\mathrm{CB}$ given the weight on the inflation gap in the Taylor rule. This stimulating monetary policy has the largest impact on output gap, which eventually turns positive.

The self-fulfilling nature of inflation expectations is exacerbated by the near-unity value of the discount factor and the flat estimated slope of the Phillips curve (see Eq. 2 and remark that the impulse response functions (IRFs) of inflation and the average inflation expectations almost perfectly overlap). This means that low inflation forecasts are almost self-fulfilling and deliver near-zero forecast errors, which allows those pessimistic inflation outlooks to survive and diffuse among the agents. ${ }^{16}$ This selection mechanism, together with expectation-driven inflation, explains the considerable persistence in inflation and inflation forecasts depicted in Figure 7. Inflation and inflation expectations cannot converge back on target until the conjugated force of positive output gaps and low interest rates become strong enough to overcome the almost self-fulfilling force of low inflation expectations. ${ }^{17}$ Those dynamics generate the inflation-less recovery. This prolonged period of positive output gaps may also suggest that the economy may really settle back to equilibrium only after full tapering by the CB.

Finally, it is interesting to note that our model reproduces another stylized fact discussed in Mankiw et al. (2003): a recession is associated with an increase in the dispersion of forecasts among agents or, in other words, the level of disagreement between

\footnotetext{
${ }^{16}$ Similar almost self-fulfilling equilibria in the inflation dynamics at the ELB are also reported in Hommes et al. (2019), who use a forecasting laboratory experiment.

${ }^{17}$ Admittedly, the number of periods before convergence back on target appears implausibly large. However, one should bear in mind that the only policy in our simple model is a Taylor rule constrained by the ELB; hence, our model abstracts from many empirically relevant dimensions of policy that would be likely to play a role in fostering the recovery. The simple structure of the model depicts inflation as almost entirely expectation-driven. It also ignores many other empirically relevant determinants of inflation, for instance investment or wage dynamics, which could also entail a quicker inflation response. Lastly, the shock that we consider is arbitrarily large and is only meant for illustrative purposes, not to match any empirical counterpart in recent history. For these reasons, one should refrain from drawing an explicit time interpretation from those IRFs.
} 
agents. $^{18}$ Indeed, Figure 7 reports how the dispersion of individual expectations spikes in the aftermath of the shock. The rise in forecast dispersion does not last: this is because of the selection pressure of the SL algorithm that pushes the agents to adapt to the 'New normal' in the aftermath of the shock. The level of heterogeneity between agents then returns to its long-run value, which is dictated by the size of the mutations.

We conclude that our simple model offers a stylized representation of the observed loss of anchorage of long-run inflation expectations depicted in Figure 1 and, more generally, of the inflation dynamics in the wake of the Great Recession and the ensuing recovery as discussed in the introduction. With this model, we offer a reading of this state of affairs as the consequence of the coordination of agents' expectations on pessimistic outlooks.

From an allocation perspective, the coordination of expectations on large and persistent recessive paths leaves out the economy into second-best equilibria with respect to the benchmark representative agent model under RE. ${ }^{19}$ Hence, SL expectations can be envisioned as a friction with respect to the $\mathrm{RE}$ representative agent allocation, which may imply a substantial welfare cost, as we now demonstrate.

\subsection{Welfare cost of social learning expectations}

How costly is the presence of expectation miscoordination in the standard two-equation NK model? To evaluate this cost, we use the welfare function, which has become the main microfounded criterion, to compare alternative policy regimes. Following Woodford (2002), we consider a second-order approximation of this criterion and use the unconditional mean to express this criterion in terms of inflation and output volatility. The detailed derivations and explicit forms are deferred to Appendix D. The corre-

\footnotetext{
${ }^{18}$ In our estimated model, the correlation between output gap and output gap forecast dispersion is significant and reaches -0.34 .

${ }^{19}$ We refer to the RE counterpart of the NK model as the first-best equilibrium because we do not study the welfare implications of the price rigidities vs. the first-best allocation under flexible prices. In this paper, our main focus is the welfare cost induced by expectation-driven cycles.
} 
sponding welfare function reads as:

$$
E\left[\mathcal{W}_{t}\right] \simeq \overline{\mathcal{W}}-\lambda^{y} E\left[\hat{y}_{t}^{2}\right]-\lambda^{\pi} E\left[\hat{\pi}_{t}^{2}\right]
$$

where $\overline{\mathcal{W}}$ is the steady-state level of welfare and $\lambda^{\pi}$ and $\lambda^{y}$ are, respectively, the elasticities of the loss function with respect to the variance of the inflation gap $E\left[\hat{\pi}_{t}^{2}\right]$ and the output gap $E\left[\hat{y}_{t}^{2}\right]$. It is straightforward to notice that macroeconomic volatility reduces the welfare of households.

While in representative-agent models the loss function is unique, it may be expressed in an agent-specific manner in a heterogeneous-agent framework. Since the aggregation of agents is performed within the linearized model, we proceed in the same way with the welfare function and linearize it up to the second order. ${ }^{20}$ The welfare criterion provides a metric to compare macroeconomic performances under SL and under RE. Comparing these two allocations results in a measurement of the welfare cost of miscoordination, which can be expressed in permanent consumption equivalents (Lucas 2003). Using a standard no-arbitrage condition between the SL and the RE allocations, the fraction of consumption $\lambda$ that SL households are willing to pay to live in an RE world solves the following conditions on utility streams:

$$
\sum_{\tau=0}^{\infty} \beta^{\tau} \frac{1}{N} \sum_{j=1}^{N} \mathcal{U}\left((1+\lambda) C_{j, t+\tau}^{S L}, H_{j, t+\tau}^{S L}\right)=\sum_{\tau=0}^{\infty} \beta^{\tau} \mathcal{U}\left(C_{t+\tau}^{R E}, H_{t+\tau}^{R E}\right)
$$

where $x_{t}^{S L}$ and $x_{t}^{R E}$ denote any endogenous variable $x$ resulting from the same sequence of shocks under the two different expectation schemes.

Table 4 compares the major business cycles statistics under RE and under SL using the estimated parameters given in Table 3. This exercise allows us to disentangle the contribution of exogenous fluctuations in the RE-NK model from those additionally

\footnotetext{
${ }^{20} \mathrm{We}$ use the same aggregation procedure for the agent-specific welfare indexes as for expectations, i.e. $E\left[\mathcal{W}_{t}\right]=\frac{1}{N} \sum_{j=1}^{N} E\left[\mathcal{W}_{j, t}\right]$.
} 


\begin{tabular}{lllccc}
\hline \hline & \multicolumn{1}{c}{ Moments } & & \multicolumn{2}{c}{ Expectations } & scheme \\
& & & & $R E$ & $S L$ \\
\cline { 1 - 4 } $\operatorname{var}\left(\hat{\pi}_{t}\right)$ & - & inflation gap variance & $0.1775(0.002)$ & $0.462(0.029)$ \\
$\operatorname{var}\left(\hat{y}_{t}\right)$ & - & output gap variance & & $14.8159(0.159)$ & $19.645(0.644)$ \\
$\Delta_{t}^{\pi}$ & - & inflation gap forecast dispersion & & - & $0.2(0.001)$ \\
$\Delta_{t}^{y}$ & - & output gap forecast dispersion & & - & $0.399(0.002)$ \\
$E\left[\mathcal{W}_{t}\right]$ & - & welfare & & $-88.099(0.001)$ & $-94.6(0.09)$ \\
$\lambda$ & - & welfare cost & - & $3.303 \%$ \\
$P\left[r_{t}=1\right]$ & - & ELB probability & $0(0)$ & $0.17(0.026)$ \\
\hline \hline
\end{tabular}

Notes: Average statistics (and standard errors between brackets) over 9,400 Monte Carlo simulations of 200 periods under SL (94 series of shocks repeated 100 times) and over the same series of shocks under RE.

Table 4: Business cycles statistics and welfare under RE and SL using estimated parameters

induced by SL.

Table 4 shows that SL expectations induce considerably more macroeconomic volatility than under RE, especially by inducing endogenous ELB episodes, as explained above. These self-fulfilling recessions substantially deteriorate the welfare of households in comparison to the RE benchmark. By contrast, under the assumption of i.i.d. shocks, the rational forecasts of inflation and output gaps boil down to their targeted values (see Section 2.2). Therefore, under RE, expectations remain anchored, self-fulfilling ELB episodes cannot occur and macroeconomic volatility is negligible.

Specifically, the resulting cost of SL expectations with respect to RE reaches up to $3.3 \%$ of permanent consumption. This welfare cost is far from negligible with respect to the real business cycle literature, especially because we have assumed CRRA preferences. $^{21}$ This cost questions the effectiveness of monetary policy based on the sole setting of the nominal interest rate and leaves room for an additional monetary policy instrument to enforce the additional objective of coordinating the private sector on fundamentals. We now explore this possibility.

\footnotetext{
${ }^{21}$ Lucas (1991) finds that the overall welfare cost of business cycles is as low as $0.05 \%$ with CRRA preferences.
} 


\section{Central bank communication}

We introduce CB communication as an additional policy instrument to help anchor expectations at the target and reduce the welfare gap between the SL and the RE regimes. We first describe how we implement communication under SL and then evaluate how it is effective at steering the economy closer to the RE allocation.

\subsection{Implementing communication in SL}

We represent communication as an announcement, which we denote by $A_{t}^{C B}$, made by the $\mathrm{CB}$ at the end of any period $t$ to the attention of the agents. In the model, this announcement concerns inflation in the next period $(t+1)$. We focus on inflation because it is the primary objective under the monetary policy regime that we consider here, i.e. an inflation targeting regime. The anchoring role of monetary policy also primarily refers to inflation expectations, while views about aggregate supply, arguably beyond the sole influence of the CB, mostly drive output gap expectations. Before turning to the determination of the announced inflation values, we first explain how an announcement can easily be integrated into the SL expectation model.

We follow Arifovic et al. (2019), albeit in a simpler game, and modify the SL algorithm as follows. In any period $t$, each agent $j$ 's output and inflation gap forecasts $\left(a_{j, t}^{y}\right.$ and $\left.a_{j, t}^{\pi}\right)$ are augmented by a third component that we denote by $\psi_{j, t}$. The component $\psi_{j, t} \in[0,1]$ stands for the probability for agent $j$ in period $t$ of incorporating the CB announcement into her inflation forecast. If she does so, her inflation forecast in $t+1$ is simply aligned with the CB announcement. Conversely, with a probability $1-\psi_{j, t}$, she ignores the announcement and proceeds as previously, i.e. she sets her inflation forecast equal to her strategy $a_{j, t}^{\pi}$. The determination of her output gap forecasts remains unchanged and equal to $a_{j, t}^{y}$.

Formally, when the CB makes announcements, the expectation formation process 
of the agents given by (8) is modified as:

$$
\begin{aligned}
& E_{j, t}^{S L}\left\{\hat{\pi}_{t+1}\right\}=\left\{\begin{array}{l}
A_{t}^{C B} \text { with probability } \psi_{j, t} \\
a_{j, t}^{\pi} \text { with probability } 1-\psi_{j, t}
\end{array}\right. \\
& E_{j, t}^{S L}\left\{\hat{y}_{t+1}\right\}=a_{j, t}^{y} .
\end{aligned}
$$

The communication-augmented inflation forecast strategy $\left\{\left(\psi_{j, t}, a_{j, t}^{\pi}\right)\right\}_{j \in J}$ undergoes the same mutation and tournament processes as the output gap forecast strategy $a_{j, t}^{y}$ (see Section 2.3). The only difference from the algorithm used so far lies in the computation of the fitness of inflation forecasts. Eq. (11) is modified so as to account for the two alternatives, i.e. either following the announcement with a probability $\psi_{j, t}$, or using her own forecast $a_{j, t}^{\pi}$ with probability $1-\psi_{j, t}$. By taking into account the CB's announcements, inflation forecast performances are then computed as:

$$
F_{j, t}^{\pi}=-\psi_{j, t} \sum_{\tau=0}^{t}\left(\rho^{\pi}\right)^{\tau}\left(\widehat{\pi}_{t-\tau}-A_{t-\tau-1}^{C B}\right)^{2}-\left(1-\psi_{j, t}\right) \sum_{\tau=0}^{t}\left(\rho^{\pi}\right)^{\tau}\left(\widehat{\pi}_{t-\tau}-a_{j, t}^{\pi}\right)^{2}
$$

where the first (resp. second) term corresponds to the discounted sum of squared forecast errors had the agent followed (resp. ignored) the announcements of the CB.

The probabilities $\left\{\psi_{j}\right\}$ can be easily interpreted as the credibility of the announcements. If agents following the announcements (i.e. agents with a relatively high value of $\psi_{j}$ ) have lower forecast errors than agents ignoring the announcements $(i . e$. agents with a relatively low value of $\psi_{j}$ ), the following strategy shall spread among agents, which means that the average value of $\psi$ across agents shall increase. The opposite shall hold if following the announcements performs more poorly than ignoring them. Thus, SL agents endogenously build trust or distrust in the communication of the CB as a function of the relative forecasting performances of each alternative.

We now consider two scenarios that differ by the announcements of the CB. 


\subsection{Two communication scenarios}

Announcement of the inflation target In the first communication scenario, the CB always announces the MSV solution associated with the socially optimal steadystate, i.e. the inflation target. We then have $A_{t}^{C B}=0$. The rationale for this first scenario is that announcing the target is the first requirement of the widespread inflation targeting regimes. Indeed, over the Great Moderation period, the inflation target has been thought of as an anchor for long-run inflation expectations. We wish to investigate this anchoring power in the context of our heterogeneous expectation environment that is subject to occasionally long-lasting ELB episodes and mimics the 'new normal'. It should be noted that the target corresponds to the RE inflation forecasts in our simple model. The announcement of the CB is therefore consistent with the conduct of monetary policy under RE. Hence, the inflation target is redundant information to RE agents, but this piece of information may play a non-trivial role under SL.

Announcement of an inflation forecast In the second communication scenario, we assume that the $\mathrm{CB}$ announces its own inflation forecasts for $t+1$ given the available information up to period $t$. The policy authority does so by estimating in every period a vector autoregressive (VAR) forecasting model. The choice of a VAR model is natural given that it is commonly used by policy makers to form forecasts (see Eusepi \& Preston (2010) for a similar assumption). It is also reasonable to assume that the CB is endowed with a more sophisticated forecasting model than the agents in light of the considerable number of resources that CBs devote to forecasting. Assuming VAR forecasting also amounts to assuming that the $\mathrm{CB}$ is aware of agents being boundedly rational and, therefore, includes past realizations of the endogenous variables in its forecasting model to account for the propagation mechanism induced by learning agents. Indeed, such a forecasting model would be misspecified should the agents have RE and, hence, the economy evolve according to the MSV solution. In this second communication scenario, 
the announcement of the $\mathrm{CB}$ is therefore consistent with the conduct of monetary policy under SL. ${ }^{22}$

The CB updates the estimates of the coefficients of its VAR model as additional observations become available using recursive least squares. Formally, let $X_{t}=\left[\hat{\pi}_{t-1}, \hat{y}_{t-1}\right.$, $\left.\cdots, \hat{\pi}_{t-\ell}, \hat{y}_{t-\ell}\right]$ be the $(2 \ell \times 1)$ vector of regressors summarizing the available information in time $t, w_{t}$ the $(2 \ell \times 2)$ vector of estimated coefficients and $z_{t}=\left(\hat{\pi}_{t}, \hat{y}_{t}\right)$ the $(1 \times 2)$ vector of new incoming observations. The constant is omitted for the VAR model to be consistent with monetary policy conducted so as to deliver zero deviations from steady-state on average. Furthermore, we use $\ell=8$, which is in line with the memory of the agents that is implied by the estimated value of the fitness memory on inflation (see, again, Table 3). ${ }^{23}$ At the end of each period $t$, the CB updates its forecasting model as:

$$
\begin{aligned}
& w_{t}=w_{t-1}+g R_{t}^{-1} X_{t}\left(y_{t}-X_{t}^{\prime} c_{t-1}\right) \\
& R_{t}=R_{t-1}+g\left(X_{t} X_{t}^{\prime}-R_{t-1}\right)
\end{aligned}
$$

where $R$ is the $(2 \ell \times 2 \ell)$ covariance matrix between the regressors and $g>0$, a (small) gain that allows the algorithm to discard distant past observations. The two-step-ahead VAR forecasts are given by $E_{t}^{C B} z_{t+1}=w_{t}^{\prime 2} z_{t}$, of which the announcement consists of the inflation forecast:

$$
A_{t}^{C B}=w_{1, t}^{\prime 2} z_{t}
$$

where $w_{1, t}^{\prime}$ refers to the first term of the vector $w^{\prime}$.

Before proceeding to the simulations, we need to specify an initial distribution of the probabilities $\left\{\psi_{j, 0}\right\}$ across agents. There is no obvious benchmark for the average

\footnotetext{
${ }^{22}$ The MSV solution under SL would be a complicated and non-linear function of all the states in the system, including those pertaining to the SL process, and an explicit form is not available. We claim that the best the $\mathrm{CB}$ can do in such an environment is to estimate the law of motion of the economy with an atheoretical model, such as a VAR.

${ }^{23}$ The results are robust to more or fewer lags and to assuming a decreasing gain.
} 
credibility of the announcements. Yet, the initial level of credibility of the target (or in other words, whether the CB has first to build credibility or whether it is already there) is likely to influence the stabilizing power of communication in the face of shocks. In the simulations below, we take a neutral stand and draw the probabilities $\left\{\psi_{j, 0}\right\}$ from a normal distribution centered around 0.5 with a standard deviation equal to 0.25 , a value that is also taken to dictate the mutation process of the probabilities $\left\{\psi_{j, 0}\right\} .{ }^{24}$

We now compare the outcomes under those two communication scenarios with those under SL and under the RE benchmark. We first examine the implications of communication on business cycles statistics and then investigate the propagation mechanism by examining the response to a shock under the three communication scenarios considered.

\subsection{Communication and macroeconomic stabilization}

We compare the business cycles statistics of the model under RE, under SL and under the two communication scenarios, namely the communication of the target and the communication of the CB's inflation forecast. ${ }^{25}$ Table 5 reports the results from the comparison exercise. For ease of reading, the first two columns recall the statistics under RE and SL obtained in Table 4 and the next two columns report those statistics when, respectively, the target and the inflation forecasts are announced. We draw two main conclusions from the table.

The first one is obtained by comparing the model under RE, under SL and under the communication scenarios as a whole, no matter whether the target or inflation forecasts are announced. The first three rows of Table 5 indicate that communication significantly improves macroeconomic stabilization with respect to the baseline heterogeneous expectation model. In particular, the volatility of inflation is divided by

\footnotetext{
${ }^{24}$ Results are robust to alternative values of the average initial credibility, e.g. as low as 0.1 and as high as 0.9. Simulations are available from the authors upon request.

${ }^{25}$ To rigorously compare the communication scenarios, we draw a large number of shocks that we feed to the model under each scenario. We repeat this procedure on different chains to obtain an asymptotic version of the business cycles statistics.
} 


\begin{tabular}{|c|c|c|c|c|}
\hline $\begin{array}{l}\text { Expectations: } \\
\text { Type of Communication }\end{array}$ & $\begin{array}{c}R E \\
\times\end{array}$ & $\begin{array}{c}S L \\
\times\end{array}$ & $\begin{array}{c}S L \\
\text { target }\end{array}$ & $\begin{array}{c}S L \\
\operatorname{VAR}(8) \text { forecast }\end{array}$ \\
\hline $\begin{array}{l}\text { Macroeconomic variability: } \\
\operatorname{var}\left(\hat{\pi}_{t}\right) \\
\operatorname{var}\left(\hat{y}_{t}\right)\end{array}$ & $\begin{array}{r}0.178(0.002) \\
14.816(0.159)\end{array}$ & $\begin{array}{r}0.463(0.029) \\
19.645(0.644)\end{array}$ & $\begin{array}{r}0.215(0.010) \\
17.181(0.310)\end{array}$ & $\begin{array}{r}0.2445(0.01) \\
17.118(0.304)\end{array}$ \\
\hline$\frac{\text { ELB occurrence: }}{P\left[i_{t}=0\right]}$ & $0.000(0.000)$ & $0.17(0.026)$ & $0.038(0.012)$ & $0.002(0.001)$ \\
\hline $\begin{array}{l}\text { Expectation dispersion: } \\
\Delta_{t}^{\pi} \\
\Delta_{t}^{y}\end{array}$ & $\begin{array}{l}x \\
\times\end{array}$ & $\begin{array}{c}0.2(0.001) \\
0.399(0.002)\end{array}$ & $\begin{array}{l}0.138(0.004) \\
0.396(0.002)\end{array}$ & $\begin{array}{l}0.155(0.0045) \\
0.397(0.002)\end{array}$ \\
\hline $\begin{array}{l}\text { Expectation anchorage: } \\
\Omega_{t}^{\pi} \\
\Omega_{t}^{y}\end{array}$ & $\begin{array}{l}0.000(0.000) \\
0.000(0.000)\end{array}$ & $\begin{array}{l}0.887\left(0.10^{r}\right) \\
10.031(1.22)\end{array}$ & $\begin{array}{l}0.108(0.02) \\
6.433(0.336)\end{array}$ & $\begin{array}{c}0.176(0.0028) \\
6.31(0.359)\end{array}$ \\
\hline $\begin{array}{l}\text { Welfare: } \\
E\left[\mathcal{W}_{t}\right] \\
\lambda(\%)\end{array}$ & $\begin{array}{c}-88.099(0.001) \\
\times\end{array}$ & $\begin{array}{c}-94.599(0.089) \\
3.303 \%\end{array}$ & $\begin{array}{c}-88.92(0.02) \\
0.411 \%\end{array}$ & $\begin{array}{c}-89.693(0.0017) \\
0.8 \%\end{array}$ \\
\hline
\end{tabular}

Notes: See Table 4.

Table 5: Business cycles statistics under RE, under SL and with CB communication about the inflation target and the inflation forecasts

more than two when the CB communicates than when it does not and the risk of ELB episodes drops considerably in the presence of $\mathrm{CB}$ communication.

A look at the next four lines of Table 5 reveals that not only are expectations better coordinated (i.e. disagreement between agents is reduced) in the presence than in the absence of communication, but coordination occurs around the CB objectives (i.e. expectations are better anchored at the target). The anchoring effect of communication operates mainly on inflation expectations as we have assumed that the CB communicates about inflation. Nevertheless, its overall stabilizing effect contributes to anchor output expectations as well through the IS equation. Hence, we conclude that in our model, CB communication acts as an anchor for heterogeneous expectations; and, by improving their coordination, communication contributes to macroeconomic stabilization. This effect translates into a narrower, yet positive, welfare gap with respect to the RE representative agent benchmark. Hence, communication does not fully eliminate the frictions associated with heterogeneous expectations but strongly and significantly 
dampens their macroeconomic effects. Those results are very much in line with Coibion et al. (2018)'s empirical work, where clear communication significantly reshapes inflation expectations and real interest rate to push aggregate demand upward.

By comparing now the two communication scenarios, we come to a second insight: performances are fairly similar. Yet, communicating the inflation target entails a lower inflation variability than communicating forecasts, which translates into a higher welfare. To explain this result, we make the following conjecture: in an environment with shocks that temporarily trigger deviations from the target, the CB forecasts may constitute a pro-cyclical signal, in the sense that off-target forecasts may turn self-confirming and contribute to drive expectations away from the target. Before concluding, we investigate this conjecture further by considering how the reaction of the model to a large unexpected shock is affected by communication.

\subsection{Propagation under alternative communications}

Figure 8 contrasts the IRFs of the estimated model to the same pessimistic shock as in Figure 7 under SL (the dotted line recalls the median realization) and under the two different communication scenarios (the blue line with triangles represents the case where the target is announced and the yellow line with squares the case where the inflation forecasts are announced). This exercise serves the purpose of illustrating how and which CB communication can act as an anchoring device for heterogeneous expectations and progressively steer expectations back on target.

Let us now discuss the propagation of the shock in the presence of communication. First, in the wake of the shock, both communication strategies result in a loss of credibility. As a consequence, both types of announcement temporarily lose their anchoring power on agents' inflation expectations. This can be easily seen by looking at Figure 7h, where credibility in the announcements invariably drops towards zero right after the shock. When announcing the target, the credibility loss stems from the actual re- 
a. Inflation gap $\hat{\pi}_{t}$

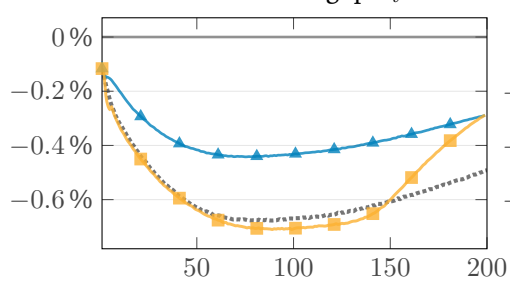

d. Output gap $\hat{y}_{t}$

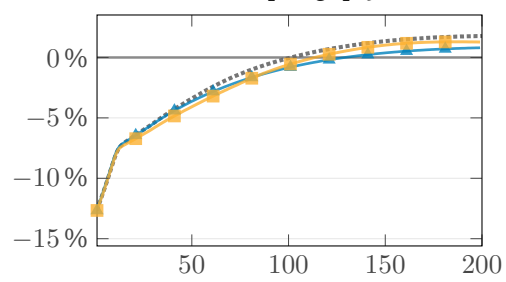

g. Central bank announcement $A_{t}^{C B}$

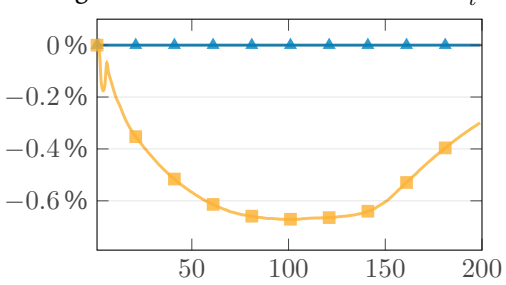

b. Mean inflation expectations $E_{t}^{S L} \hat{\pi}_{t+1} \quad$ c. Dispersion inflation expectations $\Delta_{t}^{\pi}$

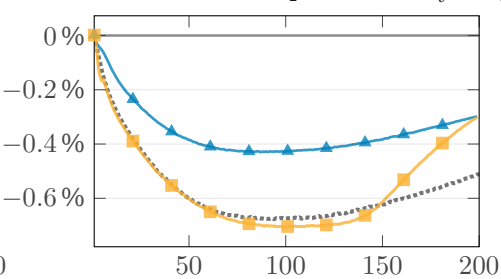

e. Mean output expectations $E_{t}^{S L} \hat{y}_{t+1}$

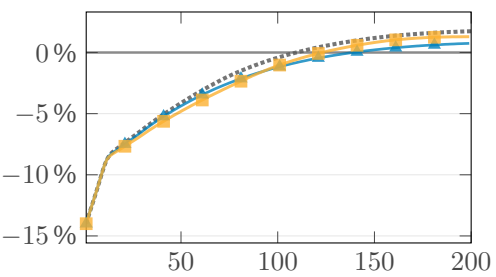

h. Average credibility rate $\psi_{t}$

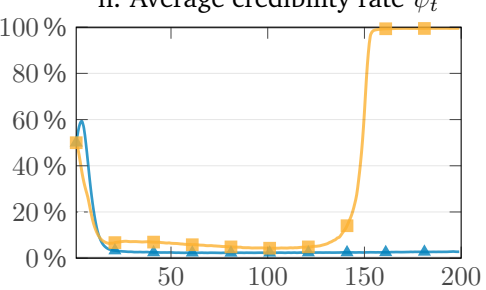

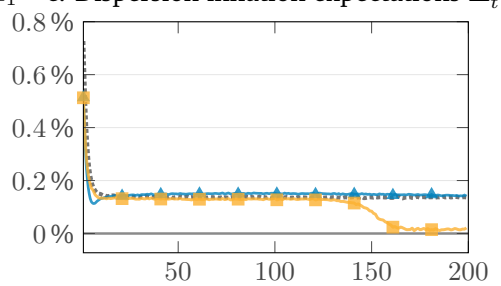

f. Dispersion output expectations $\Delta_{t}^{y}$

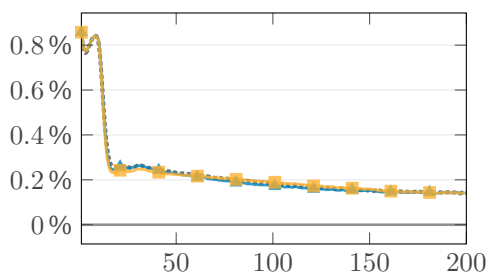

i. Dispersion of credibility rates $\Delta_{t}^{\psi}$

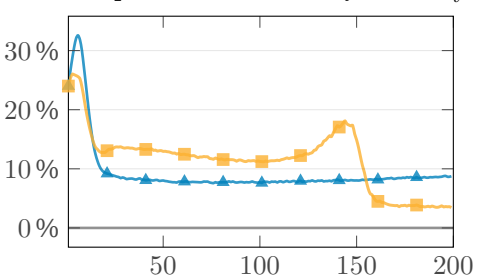

j. Nominal interest rate $\hat{i}_{t}$
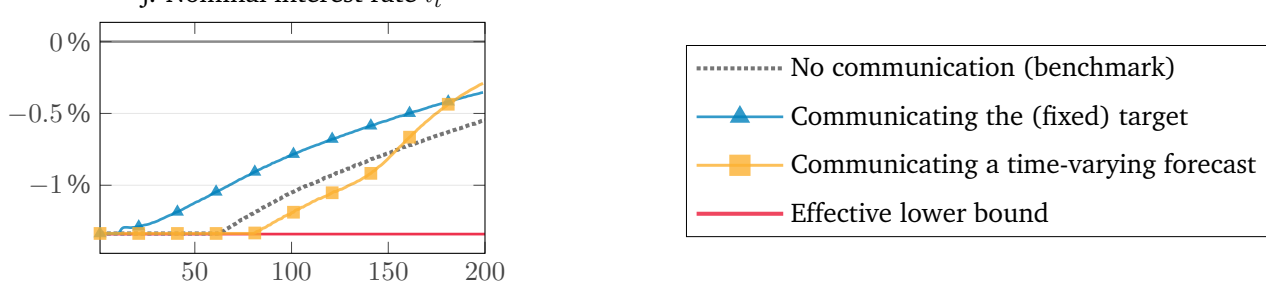

Notes: See Figure 7. The median realizations over 1,000 Monte Carlo simulations are reported. The red dashed-dotted line corresponds to the baseline SL model without communication. The blue lines report the scenarios with communication about the CB forecasts (solid line) and the target (dashed line).

Figure 8: IRFs of the estimated model to a one-period $-14 \%$ output gap expectation shock under various communication scenarios

alizations of inflation drifting away from the target. When announcing forecasts, the credibility loss results from the inaccuracy of the announced forecasts, as the pessimistic shock is unexpected - to see that, look at the discrepancy between the plunging inflation and the near-target announcements immediately after the shock (Fig. 7a vs. $7 \mathrm{~g}$ ). In both cases, the forecasting performances of the followers are worse than the ones of the non-followers who hold below-target expectations that are almost self-fulfilling (see, again, the discussion in Section 4.2). As a result of the selection pressure of the 
SL mechanism, a large fraction of the agents stop following the CB's announcements.

As a consequence, the persistence induced by the propagation mechanism under SL survives in the presence of communication and the convergence back to the target, albeit faster, is not immediate. In other words, in our model, agents need to 'see it to believe it': regardless of the communication strategy, if the CB's announcements are decoupled from the actual inflation dynamics, they lose their anchoring power on expectations and the $\mathrm{CB}$ is exposed to a risk of credibility loss.

However, as time goes forward, dynamics under the two communication scenarios start differing. In the presence of communication of inflation forecasts, the $\mathrm{CB}$, by updating its model, provides more accurate forecasts - to see that, notice the similarity between the announcements and actual inflation some periods after the shock. As a result of the selection pressure of SL, the $\mathrm{CB}$ starts regaining credibility - notice the average credibility $\psi$ going back to one after a bit less than 150 periods under that scenario, which is not the case when communication concerns the target. By contrast, when announcing the target, the $\mathrm{CB}$ can only regain its credibility once the system has converged back to the steady-state and the actual realizations of inflation are back in line with the target announcements, which may take a considerable amount of time, as discussed in Section 4.

Furthermore, the IRFs of the forecast dispersion clearly show how coordination on the forecast announcements leads to a reduction in expectation heterogeneity, which is the main driver of macroeconomic dynamics under SL - to see that, notice the drop in inflation forecast dispersion (Fig. 7c) as the average probability of following the announcements $\psi$ reaches one (Fig. $7 \mathrm{~h}$ ). As a result of that coordinating effect of the expectations, it is striking to see that convergence back to the target is faster in the presence of forecast communication than in the absence of communication or when the $\mathrm{CB}$ communicates about the target.

Yet, communicating forecasts is not a panacea: it also accentuates the downturn. 
Indeed, inflation dives deeper, the ELB binds for a longer period (Fig. 7j) and output overshoots further (Figs. 7d-7e) when the CB announces its forecasts than when it announces its target. This effect arises because the announced forecasts extrapolate the bust and turn self-confirming per the self-fulfilling nature of expectations in the model. This effect is striking from the three graphs of the average inflation forecasts (Fig. 7b), the CB forecast announcements (Fig. 7g) and the actual inflation (Fig. 7a) which all almost overlap. Hence, following or discarding the announcements yields to the same forecasts and the same prediction errors. This observation is fully in tune with the conjecture made in Section 5.2 of higher inflation volatility and lower welfare when the forecasts rather than the target are communicated.

By contrast, when the $\mathrm{CB}$ communicates about the target, the initial drop in inflation and inflation expectations is milder than when the CB communicates forecasts or does not communicate at all; this is because a fraction of the agents have initially anchored their inflation expectations to the target. As a result, the ELB duration is

shortest under that scenario but the shock remains particularly persistent - this is a direct implication from our analysis in Figure $6 \mathrm{~b}$.

\section{Conclusion}

This paper develops a model that features expectation-driven business cycles. The key mechanism works through heterogeneous expectations that may lose their anchorage to the central bank target and persistently coordinate on below-target paths, which triggers prolonged ELB episodes. Heterogeneous expectations are introduced via an SL process into an otherwise standard two-equation macroeconomic model with a constrained Taylor rule. Our model nests the RE representative agent benchmark. In particular, we use white noise fundamental shocks to identify the propagation mechanism stemming from expectations in the formation of business cycles. 
Our first contribution is to bring such a model to the data and estimate jointly its fundamental and learning parameters by matching moments from both US inflation and output gaps and the SPF. Our parsimonious model is able to account for ten stylized facts, including properties related to heterogeneity in forecasts, persistence in macroeconomic variables and endogenous occurrence of ELB episodes.

We then analyze the dynamics of the model and show that the basin of attraction of the target under SL is larger than the determinacy region under RE. In this context, ELB episodes are episodes where heterogeneous expectations have coordinated on pessimistic outlooks following a bad series of fundamental shocks and have visited regions of that basin from where the transition back on the target is particularly slow. Our second major contribution is then to provide a framework that has the ability to account for the inflation dynamics observed over the last decade in the US and in the Euro area that are challenging to capture in standard macroeconomic models. In particular, our model accounts for the 'missing disinflation' along the Great Recession per its stable but below-target dynamics in inflation and inflation expectations and extensive ELB episodes. It also accounts for the 'inflation-less recovery' and offers an interpretation of that phenomenon as the consequence of the combination of unanchored, below-target inflation expectations that put downward pressure on inflation and the boosting effect of below-target interest rates on output.

As a third contribution, we quantify the welfare cost of heterogeneous and learning expectations with respect to the $\mathrm{RE}$ benchmark and introduce $\mathrm{CB}$ communication to help mitigate that cost. Precisely, the $\mathrm{CB}$ announces its inflation target or its inflation forecasts, and agents choose to anchor their expectations to the announcements depending on the relative forecast performances of their own forecast strategy versus the CB announcements. Therefore, the credibility of the announcements is endogenous and follows the same evolutionary process as the forecasts of the agents. We show that communication, no matter the content, helps anchor expectations around the socially 
desirable state and decreases the likelihood of expectation-driven recessions at the ELB.

However, in the face of a large unexpected shock, the CB invariably loses credibility when announcing the inflation target as inflation plunges. As a result, the target announcements barely affect the persistence of the shock. On the other hand, communication of the inflation forecasts helps mitigate the bust and accelerate the recovery, but the pessimistic CB forecasts turn self-confirming and accentuate the drop in inflation and the length of the ELB episodes with respect to the scenario where the CB communicates the target.

Finally, our model offers a simple framework that yet opens up the possibility for analyzing a rich set of monetary policy alternatives. As for our estimation routine, it may be applied to a wide range of standard workhorse models that could then be explored under heterogeneous expectations. Those research avenues are left for further work.

\section{References}

Adam, K. \& Marcet, A. (2011), 'Internal rationality, imperfect market knowledge and asset prices', Journal of Economic Theory 146(3), 1224-1252.

Angeletos, G.-M., Collard, F. \& Dellas, H. (2018), 'Quantifying confidence', Econometrica 86(5), 1689-1726.

Anufriev, M. \& Hommes, C. (2012), 'Evolutionary selection of individual expectations and aggregate outcomes in asset pricing experiments', American Economic Journal: Microeconomics 4(4), 35-64.

Arifovic, J., Boitnott, J. F. \& Duffy, J. (2019), 'Learning correlated equilibria: An evolutionary approach', Journal of Economic Behavior $\&$ Organization 157(C), 171190.

Arifovic, J., Bullard, J. \& Kostyshyna, O. (2013), 'Social learning and monetary policy rules', The Economic Journal 123(567), 38-76.

Arifovic, J. \& Ledyard, J. (2012), 'Individual evolutionary learning, other-regarding preferences, and the voluntary contributions mechanism', Journal of Public Economics 96(9), 808-823. 
Arifovic, J., Schmitt-Grohé, S. \& Uribe, M. (2018), 'Learning to live in a liquidity trap', Journal of Economic Dynamics and Control 89, 120-136.

Aruoba, B., Cuba-Borda, P. \& Schorfheide, F. (2017), 'Macroeconomic dynamics near the ZLB: A tale of two countries', The Review of Economic Studies 85(1), 87-118.

Ascari, G. \& Sbordone, A. M. (2014), 'The macroeconomics of trend inflation', Journal of Economic Literature 52(3), 679-739.

Benhabib, J., Schmitt-Grohé, S. \& Uribe, M. (2001), 'The perils of Taylor rules', Journal of Economic Theory 96(1), 40-69.

Blanchard \& Kahn (1980), 'The solution of linear difference models under rational expectations', Econometrica 48(5), 1305-1311.

Branch, W. A. (2004), 'The theory of rationally heterogeneous expectations: Evidence from survey data on inflation expectations', The Economic Journal 114(497), 592621.

Branch, W. \& Evans, G. (2007), 'Model uncertainty and endogenous volatility', Review of Economic Dynamics 10(2), 207-237.

Bullard, J. \& Mitra, K. (2002), 'Learning about monetary policy rules', Journal of Monetary Economics 49(6), 1105-1129.

Carroll, C. D. (2003), 'Macroeconomic expectations of households and professional forecasters', Quarterly Journal of economics 118(1), 269-298.

Cavallo, A., Cruces, G. \& Perez-Truglia, R. (2017), 'Inflation expectations, learning, and supermarket prices: Evidence from survey experiments', American Economic Journal: Macroeconomics 9(3), 1-35.

Coibion, O., Gorodnichenko, Y., Kumar, S. \& Pedemonte, M. (2018), Inflation expectations as a policy tool?, Technical Report w24788, National Bureau of Economic Research.

Coibion, O., Gorodnichenko, Y. \& Weber, M. (2019), Monetary policy communications and their effects on household inflation expectations, Technical report, National Bureau of Economic Research.

Del Negro, M. \& Eusepi, S. (2011), 'Fitting observed inflation expectations', Journal of Economic Dynamics and Control 35(12), 2105-2131.

Eusepi, S. \& Preston, B. (2010), 'Central bank communication and expectations stabilization', American Economic Journal: Macroeconomics 2(3), 235-271.

Evans, G. W., Guse, E. \& Honkapohja, S. (2008), 'Liquidity traps, learning and stagnation', European Economic Review 52(8), 1438-1463. 
Galí, J. (2015), Monetary policy, inflation, and the business cycle: An introduction to the New Keynesian framework and its applications, Princeton University Press.

Galí, J. (2018), 'The state of New Keynesian Economics: A partial assessment', Journal of Economic Perspectives 32(3), 87-112.

Gourio, F., Kashyap, A. K. \& Sim, J. W. (2018), 'The trade offs in leaning against the wind', IMF Economic Review 66(1), 70-115.

Guerrieri, L. \& Iacoviello, M. (2015), 'Occbin: A toolkit for solving dynamic models with occasionally binding constraints easily', Journal of Monetary Economics 70, 2238.

Hansen, N., Müller, S. D. \& Koumoutsakos, P. (2003), 'Reducing the time complexity of the derandomized evolution strategy with covariance matrix adaptation (CMA-ES)', Evolutionary Computation 11(1), 1-18.

Hommes, C. (2011), 'The heterogeneous expectations hypothesis: Some evidence from the lab', Journal of Economic Dynamics and Control 35(1), 1-24.

Hommes, C. \& Lustenhouwer, J. (2019), 'Managing heterogeneous and unanchored expectations: A monetary policy analysis', Journal of Monetary Economics 107, 48 $-62$.

Hommes, C., Massaro, D. \& Salle, I. (2019), 'Monetary and fiscal policy design at the zero lower bound: Evidence from the lab', Economic Inquiry 57(2), 1120-1140.

Jarociński, M. \& Maćkowiak, B. (2018), 'Monetary-fiscal interactions and the euro area's malaise', Journal of International Economics 112, 251-266.

Jo, S. \& Sekkel, R. (2019), 'Macroeconomic uncertainty through the lens of professional forecasters', Journal of Business \& Economic Statistics 37(3), 436-446.

Lucas, R. E. (1991), Models of Business Cycles, Wiley-Blackwell.

Lucas, R. E. (2003), 'Macroeconomic priorities', American Economic Review 93(1), 114.

Malmendier, U. \& Nagel, S. (2016), 'Learning from inflation experiences', The Quarterly Journal of Economics 131(1), 53-87.

Mankiw, N. G., Reis, R. \& Wolfers, J. (2003), 'Disagreement about inflation expectations', NBER Macroeconomics Annual 18, 209-248.

McFadden, D. (1989), 'A method of simulated moments for estimation of discrete response models without numerical integration', Econometrica 57(5), 995-1026.

Mertens, T. M. \& Williams, J. C. (2019), Tying down the anchor: Monetary policy rules and the lower bound on interest rates, Technical Report 2019-14, Federal Reserve Bank of San Francisco. 
Milani, F. (2007), 'Expectations, learning and macroeconomic persistence', Journal of Monetary Economics 54(7), 2065-2082.

Nakov, A. (2008), 'Optimal and simple monetary policy rules with zero floor on the nominal interest rate', International Journal of Central Banking 4(2), 73-127.

Rossi, B. \& Sekhposyan, T. (2015), 'Macroeconomic uncertainty indices based on nowcast and forecast error distributions', American Economic Review 105(5), 650-55.

Ruge Murcia, F. J. (2007), 'Methods to estimate dynamic stochastic general equilibrium models', Journal of Economic Dynamics and Control 31(8), 2599-2636.

Schmitt-Grohé, S. \& Uribe, M. (2004), 'Optimal fiscal and monetary policy under sticky prices', Journal of Economic Theory 114(2), 198-230.

Schmitt-Grohé, S. \& Uribe, M. (2017), 'Liquidity traps and jobless recoveries', American Economic Journal: Macroeconomics 9(1), 165-204.

Slobodyan, S. \& Wouters, R. (2012a), 'Learning in a medium-scale DSGE model with expectations based on small forecasting models', American Economic Journal: Macroeconomics 4(2), 65-101.

Slobodyan, S. \& Wouters, R. (2012b), 'Learning in an estimated medium-scale DSGE model', Journal of Economic Dynamics and Control 36(1), 26-46.

Smets, F. \& Wouters, R. (2007), 'Shocks and frictions in US business cycles: A Bayesian DSGE approach', American Economic Review 97, 586-606.

Woodford, M. (2002), 'Inflation stabilization and welfare', The B.E. Journal of Macroeconomics 2(1), 1-53.

Woodford, M. (2003), Interest and prices: Foundations of a theory of monetary policy, Princeton University Press.

Woodford, M. (2013), 'Macroeconomic analysis without the rational expectations hypothesis', Annual Review of Economics 5(1), 303-346. 


\section{A Solution under rational expectations}

We solve the model under RE using the method of undetermined coefficients (with and without the ELB).

First, inserting Eq. (5) into Eq. (1) provides the reduced-form expression of the log-linearized model:

$$
z_{t}=\alpha+B E_{t} z_{t+1}+\chi^{g} \widehat{g}_{t}+\chi^{u} \widehat{u}_{t}
$$

with the two endogenous variables $z_{t}=\left(\widehat{y}_{t} \widehat{\pi}_{t}\right)^{\prime}$; matrices $\chi^{g}=\left(\begin{array}{ll}1 & \kappa\end{array}\right)^{\prime}$ and $\chi^{u}=\left(\begin{array}{ll}0 & 1\end{array}\right)^{\prime}$ are related to the shocks $g$ and $u$ while $\alpha$ and $B$ are related, respectively, to the steadystate values and the forward-looking variables. The values of $\alpha$ and $B$ depend on the steady-state considered.

Given Eq. (17), the general form of the MSV solution reads as:

$$
z_{t}=a+c \widehat{g}_{t}+d \widehat{u}_{t}
$$

where the coefficient values in matrices $a, c$ and $d$ depend on whether the ELB is binding or not.

Taking expectations based on the specification of the stochastic processes (3) yields (assuming for now that shocks are observable in $t$ ):

$$
E\left(z_{t+1}\right)=a+c \rho^{g} \widehat{g}_{t}+d \rho^{u} \widehat{u}_{t} .
$$

Inserting Eq. (19) back into (17) uniquely identifies the MSV solution as:

$$
z_{t}=\alpha+B a+g_{t}\left(B c \rho^{g}+\chi^{g}\right)+u_{t}\left(B d \rho^{u}+\chi^{u}\right),
$$

with $a=(I-B)^{-1} \alpha, c=\left(I-B \rho^{g}\right)^{-1} \chi^{g}$ and $d=\left(I-B \rho^{u}\right)^{-1} \chi^{u}$, which makes clear that the coefficient values of matrices $B$ and $\alpha$ depend on the steady-state considered.

First, we consider the REE at the targeted steady-state that we denote by a star superscript. We insert the specification of the Taylor rule (5) when the ELB is not binding, i.e. $\widehat{\imath_{t}}=\phi^{\pi} \mathbb{E}_{t} \widehat{\pi}_{t+1}+\phi^{y} \mathbb{E}_{t} \widehat{y}_{t+1}$ into (1) and obtain the expressions: $\alpha^{T}=\left[\begin{array}{l}0 \\ 0\end{array}\right]$ and $B^{T}=\left[\begin{array}{cc}1-\sigma^{-1} \phi^{y} & \sigma^{-1}\left(1-\phi^{\pi}\right) \\ \kappa\left(1-\sigma^{-1} \phi^{y}\right) & \beta+\sigma^{-1}\left(1-\phi^{\pi}\right) \kappa\end{array}\right]$.

The MSV-REE solution at the target is then given by:

$$
a^{T}=\left(I-B^{T}\right)^{-1} \alpha^{T}, c^{T}=\left(I-B^{T} \rho^{g}\right)^{-1} \chi^{g} \text { and } d^{T}=\left(I-B^{T} \rho^{u}\right)^{-1} \chi^{u} .
$$

Similarly, when the ELB is binding, the monetary policy rule reads as $\widehat{t}_{t}=-\bar{r}$. Inserting this expression back into Eq. 1, the REE at the ELB, which we denote with a elb superscript, is described by:

$$
\alpha^{e l b}=\left[\sigma^{-1} \bar{r}, \kappa \sigma^{-1} \bar{r}\right] \text { and } B^{e l b}=\left[\begin{array}{cc}
1 & \sigma^{-1} \\
\kappa & \beta+\sigma^{-1} \kappa
\end{array}\right] .
$$


If shocks are i.i.d., $\rho^{g}=\rho^{u}=0$, Eq. (19) reduces to an intercept $a$, with $a^{T}=$ $\left(I-B^{T}\right)^{-1} \alpha^{T}$ at the target and $a^{e l b}=\left(I-B^{e l b}\right)^{-1} \alpha^{e l b}$ at the ELB.

\section{B Determinacy and E-stability}

The REE (21) is determinate under RE if the two eigenvalues of matrix $B^{T}$ lie within the unit circle. This is the case if all three conditions $\phi^{y}<\sigma\left(1+\beta^{-1}\right), 0<\kappa\left(\sigma^{\pi}-\right.$ $1)+(1+\beta) \sigma^{y}<2 \sigma(1+\beta)$ and $\kappa\left(\phi^{\pi}-1\right)+(1-\beta) \phi^{y}>0$ hold (Bullard \& Mitra 2002, p. 1121). Our calibration imposes these restrictions on the parameters values. Specifically, the REE values at the target are $a^{T}=(00)^{\prime}$, and the REE is determinate (the two eigenvalues are complex and equal $\lambda_{i}^{-}=.933-.027 i$ and $\lambda_{i}^{+}=.933+.027 i$ ). Note that the same conditions ensure that this solution is E-stable, i.e. stable if agents use adaptive learning instead of RE (Bullard \& Mitra 2002).

By contrast, the REE at the ELB (22) is indeterminate under RE and unstable under learning. To see that, notice that the characteristic polynomial of $B^{\text {elb }}$ is $\beta+\lambda(-1-\beta-$ $\left.\kappa \sigma^{-1}\right)+\lambda^{2}=0 \Leftrightarrow a_{0}+a_{1} \lambda+\lambda^{2}$. For both eigenvalues to be within the unit circle and the REE to be determinate, we need $\left|a_{0}\right|<1$ and $\left|a_{1}\right|<1+a_{0}$. The first condition always holds as $\beta<1$ but the second is always violated as $\sigma^{-1} \kappa>0$. Therefore, the deflationary state is indeterminate under RE and features multiple equilibria. ${ }^{26}$

Furthermore, the determinant of $B^{e l b}-I$ ( $I$ being the identity matrix) is $-\sigma^{-1} \kappa<0$, which implies that one eigenvalue of $B^{e l b}-I$ has negative real part and one has positive real part (equivalently, one eigenvalue of $B^{e l b}$ is lower than one, the other is not). Therefore, under learning, the deflationary steady-state is unstable and is a saddle.

Under our calibration, the REE values at the ELB are $a^{e l b}=(-0.007-0.013)^{\prime}$, and the two eigenvalues of $B^{e l b}$ are real and equal $\lambda_{i}^{-}=0.906<1$ and $\lambda_{i}^{+}=1.099>1$.

\section{Estimation strategy}

We proceed by following the related literature on the estimation of macroeconomic models under RE, but we need to adapt the method to a model under SL because SL introduces an additional non-linearity and an additional source of stochasticity into the model. This section explains how we do so.

Together with the specification of the SL process, the model - described in Eq. 1 to 5 - can be expressed in the following compact form:

$$
\mathbb{E}_{t}^{S L}\left\{f_{\Theta}\left(z_{t+1}, z_{t}, \varepsilon_{t}\right)\right\}=0
$$

where $z_{t}$ is the set of endogenous variables, $\varepsilon_{t}$ the set of $i . i . d$. shocks and $f_{\Theta}(\cdot)$ the equations of the model using calibration $\Theta$.

First, we partition the parameters $\Theta$ into two sets: the first set contains mainly monetary policy and preferences parameters, which we calibrate following the literature

\footnotetext{
${ }^{26}$ Another way to see that is to note that the ELB corresponds to an interest rate peg as described in Woodford (2003, Chap. 2) that gives rise to indeterminacy.
} 
as given in Table 1 . The second set, $\theta \in \Theta$, contains parameters that we estimate by minimizing the distance between simulated and empirical moments.

To do so, the first difference that we need to address between an RE and an SL specification of the model is the issue of stochastic replications. An RE model only involves stochasticity in the exogenous shocks ( $\epsilon$ in Eq. (23)). Hence, the standard way to proceed is to exploit the asymptotic properties of Monte Carlo methods: a high number $S$ of time series (also called 'chains') of shocks is drawn and the average over the whole series is used to obtain an unconditional measure of the simulated moments.

By contrast, the SL model introduces an additional source of stochasticity via the mutation processes and the random pairing in the tournament selection. We therefore have to apply Monte Carlo methods on each chain of shocks $s \in S$ to obtain a representative behavior of the model under SL for any given chain $s$ of shocks.

Specifically, we draw $S=100$ chains of shocks $u$ and $g$ at the beginning of the estimation procedure and keep them unchanged. For each of the 100 chains, we run 100 Monte Carlo simulations of the model under SL and only retain one 'representative' simulation prior to the estimation exercise. To select this representative simulation, we choose the one for which the squared distances of inflation and output gaps to their median values over the 100 replications is the smallest. ${ }^{27}$ Therefore, for each chain of shocks, we retain only one simulation. We do so for each of the $S=100$ series of shocks. This has the advantage of considerably reducing the computational cost of the estimation and is analogous to choosing the stable root in the policy function of an RE model, as commonly used in the standard related literature.

Let us now define $m_{T}\left(x_{t}\right)$, a $p \times 1$ vector of moments calculated using stationary and ergodic real data $x_{t}$ of sample size $T$, and $m_{s, \tau}\left(\hat{x}_{t}^{\theta}\right)$, the model-generated counterpart based on artificial series $\hat{x}_{t}^{\theta}$ of size $\tau$ generated using the set of parameters $\theta$ and Eq. (23). In our case, $p=10$ as we match 10 moments. Our estimation procedure aims to minimize the distance between those two sets of moments.

To do so, the two sets of data must have similar properties. In particular, it is best practice to consider ergodic simulated data and same-sized samples. We then use $T=200$ (quarters) given the time span considered in the SPF data. As for the ergodicity, we note that RE models are usually ergodic, i.e. the generated moments remain of similar magnitude across different chains and different starting values, but nothing guarantees that such a property holds a priori under SL. Prior to estimating the model, we have checked that it is the case if we use $T=\tau=200$. Yet, we have observed that for six chains of shocks out of the 100 used, the model may be subject to unstable recessive paths. We therefore discard those six chains from the computation of the moments.

Finally, we follow Ruge Murcia (2007) and include priors on the distribution of the parameter values. We denote by $\mathcal{P}(\theta)$ those priors into the objective function. We use techniques from Bayesian econometrics based on the optimization of a log-likelihood function. We log-linearize the contribution of the priors so that the objective function

\footnotetext{
${ }^{27}$ Note that in a one-dimensional problem, this procedure boils down to selecting the median. However, as we estimate a two-dimensional model (inflation and output gaps), our procedure provides a way to approximate the median simulation.
} 
is the sum of the squared distance of the moments plus the sum of the log-priors. The resulting SMM estimator is then defined as:

$$
\hat{\theta}_{S M M}=\arg \min _{\theta}\left[m_{T}\left(x_{t}\right)-m_{s, \tau}\left(\hat{x}_{t}^{\theta}\right)\right]^{\prime} W\left[m_{T}\left(x_{t}\right)-m_{s, \tau}\left(\hat{x}_{t}^{\theta}\right)\right]+\Xi \mathcal{P}(\theta),
$$

where $m_{T}\left(x_{t}\right)-m_{s, \tau}\left(\hat{x}_{t}^{\theta}\right)$ is the distance vector between the observed and the simulated moments that we seek to minimize, as explained above, and $W$ is the weighting matrix. Hence, the matrix product in Eq. (24) provides the sum of the squares of the residuals between the observed and matched moments. The second term $\Xi \mathcal{P}(\theta)$ introduces a penalty into the objective function when the estimated values range outside their prior distributions. We set the weight on those priors to $\Xi=0.001$. This small value is necessary when using the SMM method because this method relies on a small number of observations (i.e. a small sample of the moments of the time series), which magnifies the contribution of the prior information to the objective function.

Specifically, we define a prior for $\kappa$ that is in line with empirical results by Smets \& Wouters (2007) with a Beta distribution of 0.05 mean and 0.1 standard deviation. As for the SL parameters, we impose a Beta distribution for the mutation probabilities $\mu^{x}$ and the fitness persistence $\rho^{x}, x=\{\pi, y\}$ with a prior mean and standard deviation in line with the values used in the SL literature (Arifovic et al. 2013). We further impose to the sizes of mutation $\xi^{x}$ a positive support with a diffuse prior through an inverse gamma distribution with mean 0.1 and standard deviation of 5 . These are the prior values reported in Table 3.

We solve Eq. (24) using the CMAES optimization algorithm of Hansen et al. (2003). The CMAES algorithm is a global estimation strategy that has the ability to deal with large-scale optimization problems and avoid local mimima. This algorithm provides an accurate measure of the Hessian matrix, even in the presence of bound restrictions and priors for control variables, as is the case in Eq. (24). Specifically, learning the covariance matrix in the CMAES is analogous to learning the inverse Hessian matrix in a gradient-based, local optimization method such as the quasi-Newton method.

\section{Welfare criterion}

In this section, we develop the approximation of the welfare criterion.

\section{D.1 The welfare in terms of output and prices}

Before approximating the welfare function, we first rewrite the welfare function by expressing the utility function in terms of output and price equivalents. Recall that:

$$
\begin{gathered}
\mathcal{W}_{t}=\sum_{\tau=0}^{\infty} \beta^{\tau} \mathcal{U}\left(C_{t+\tau}, H_{t+\tau}\right), \\
\text { with } \mathcal{U}\left(C_{t}, H_{t}\right)=\log \left(C_{t}\right)-\frac{\chi}{1+\phi} H_{t}^{1+\phi} .
\end{gathered}
$$


In the absence of physical capital, the resource constraint reduces to:

$$
\int_{0}^{1} y_{i t} \mathrm{~d} i=Y_{t}=C_{t}
$$

where aggregate production is the sum of the production of each variety $i$ of goods in the economy. This equation allows us to substitute output for consumption in the utility function. Using a constant-return-to-scale production function allows us to further substitute output for the hours worked. We then have $y_{i t}=h_{i t}$ and, at the aggregate, $Y_{t}=H_{t}$.

Let us now aggregate the optimal demands for each variety $i$ :

$$
\int_{0}^{1}\left(\frac{p_{i t}}{P_{t}}\right)^{-\epsilon} Y_{t} \mathrm{~d} i
$$

where $\epsilon>1$ is the elasticity of substitution between differentiated types of goods $i, p_{i t}$ is the price of variety $i$ and $P_{t}$ the aggregate price level of all varieties in the economy. Market clearing imposes (28) to be equal to (27).

In Eq. (28), the term $\int_{0}^{1}\left(\frac{p_{i t}}{P_{t}}\right)^{-\epsilon} d i$ is the price dispersion across varieties $i$ induced by price stickiness, which we rewrite as $\Delta_{t}$. To summarize, market clearing implies:

$$
H_{t}=\Delta_{t} Y_{t}
$$

Substituting consumption $C_{t}$ and labor $H_{t}$ into the utility function using expressions (27) and (29), we have:

$$
\mathcal{U}\left(Y_{t}, \Delta_{t} Y_{t}\right)=\log \left(Y_{t}\right)-\frac{\chi}{1+\phi}\left(\Delta_{t} Y_{t}\right)^{1+\phi},
$$

where $\chi=(\epsilon-1) / \epsilon$ is the inverse of the markup in the economy.

The price dispersion $\Delta_{t}$ is hard to interpret and has no observable counterpart in macroeconomic time series. Following Woodford (2003), we now express price dispersion in term of inflation.

\section{D.2 Price dispersion}

The price dispersion is induced by the Calvo probability $\theta$ that constrains firms in updating their price. Following Schmitt-Grohé \& Uribe (2004), we can rewrite the price dispersion as:

$$
\begin{aligned}
\Delta_{t} & =\int_{0}^{1}\left(\frac{P_{i t}}{P_{t}}\right)^{-\epsilon} \mathrm{d} i \\
& =(1-\theta)\left(\frac{P_{t}^{*}}{P_{t}}\right)^{-\epsilon}+\theta \Delta_{t-1} \pi_{t}^{\epsilon} \bar{\pi}^{-\epsilon},
\end{aligned}
$$

where $\bar{\pi}$ is the rate of inflation at the steady-state.

Now that we have an expression for the price dispersion, we need to replace the 
optimal price $P_{t}^{*} / P_{t}$ from the previous expression by the inflation rate. To do so, we use the aggregation condition on prices of constrained firms, and firms that can update their price approximated using the law of large numbers:

$$
\begin{aligned}
P_{t}^{1-\epsilon} & =\int_{0}^{1} P_{i t}^{1-\epsilon} d i \\
P_{t}^{1-\epsilon} & =\theta\left(\bar{\pi} P_{t-1}\right)^{1-\epsilon}+(1-\theta)\left(P_{t}^{*}\right)^{1-\epsilon} .
\end{aligned}
$$

Dividing by $P_{t}^{1-\epsilon}$, the price index becomes:

$$
1=\theta\left(\frac{\pi_{t-1}}{\bar{\pi}}\right)^{\epsilon-1}+(1-\theta)\left(\frac{P_{t}^{*}}{P_{t}}\right)^{1-\epsilon}
$$

From the latter expression, the relative optimal price $\frac{P_{t}^{*}}{P_{t}}$ is a non-linear function of past inflation:

$$
\frac{P_{t}^{*}}{P_{t}}=\left(\frac{1-\theta \pi_{t-1}^{\epsilon-1} \bar{\pi}^{1-\epsilon}}{(1-\theta)}\right)^{1 /(1-\epsilon)}
$$

Combining Eq. 35 and Eq. 36, the price dispersion term $\Delta_{t}$ may be expressed in terms of inflation:

$$
\Delta_{t}=(1-\theta)^{\frac{\epsilon}{\epsilon-1}}\left(1-\theta \pi_{t-1}^{\epsilon-1} \bar{\pi}^{1-\epsilon}\right)^{1 /(1-\epsilon)}+\theta \Delta_{t-1}\left(\frac{\pi_{t}}{\bar{\pi}}\right)^{\epsilon}
$$

In this latter expression, $\Delta_{t}$ is a function of inflation rates in $t$ and $t-1$ as well as previous dispersion $\Delta_{t-1}$. It is not possible to obtain a closed-form solution of the price dispersion as a function of inflation. However, up to second order, the variance is unconditional and we may express the variance of the price dispersion as a function of inflation (see Woodford (2003)).

\section{D.3 Approximation up to second order}

We normalize to one the hours worked, $\bar{H}=1$, which, through the production function, normalizes to one the production, i.e. $\bar{Y}=\bar{H}=1$, which also normalizes the consumption through the resources constraint, i.e. $\bar{C}=\bar{Y}=1$. In addition, since the following exercise does not aim to determine the optimal rate of inflation, we simply normalize to one the price dispersion term $\bar{\Delta}=1$ and make it independent of the steady-state inflation rate.

Let us first consider the approximation of the welfare utility function in Eq. (30). The first term in the left-hand side up to second order reads as:

$$
\begin{aligned}
\log \left(Y_{t}\right) & \simeq \log (\bar{Y})+\frac{1}{\bar{Y}}\left(Y_{t}-\bar{Y}\right)-\frac{1}{2} \frac{1}{\bar{Y}^{2}}\left(y_{t}-\bar{Y}\right)^{2} \\
& \simeq \log (\bar{Y})+\left(\hat{y}_{t}+\frac{1}{2} \hat{y}_{t}^{2}\right)-\frac{1}{2} \hat{y}_{t}^{2} \\
& \simeq \log (\bar{Y})+\hat{y}_{t}
\end{aligned}
$$

where $\hat{y}_{t}=\left(Y_{t}-\bar{Y}\right) / \bar{Y}$. Since there is no second-order term, the unconditional mean 
of the utility function on consumption is simply zero:

$$
E\left[\log \left(Y_{t}\right)\right] \simeq 0
$$

This is because the unconditional mean of any normally distributed random variable $x_{t}$ allows us to eliminate the first-order terms that are zero asymptotically, i.e. $E\left[x_{t}\right] \simeq 0$. Concerning the second term of the left-hand side of Eq. (30), up to second order, we have:

$$
\begin{gathered}
\frac{\chi}{1+\phi}\left(\Delta_{t} Y_{t}\right)^{1+\phi} \simeq \frac{\chi}{1+\phi}(\bar{Y} \bar{\Delta})^{1+\phi}+\frac{\chi}{h}(\bar{Y} \bar{\Delta})^{1+\phi}\left(Y_{t}-\bar{Y}\right)+\frac{\phi}{2} \frac{\chi}{\bar{Y}^{2}}(\bar{Y} \bar{\Delta})^{1+\phi}\left(\bar{Y}_{t}-\bar{Y}\right)^{2} \\
+\frac{\chi}{\bar{\Delta}}(\bar{Y} \bar{\Delta})^{1+\phi}\left(\Delta_{t}-\bar{\Delta}\right)+\frac{\phi}{2} \frac{\chi}{\bar{\Delta}^{2}}(\bar{Y} \bar{\Delta})^{1+\phi}\left(\Delta_{t}-\bar{\Delta}\right)^{2}
\end{gathered}
$$

With the normalization previously introduced, and recalling that the parameter $\chi$ is the inverse of the markup, we have:

$$
\frac{\chi}{1+\phi}\left(\Delta_{t} Y_{t}\right)^{1+\phi} \simeq \frac{\epsilon-1}{\epsilon}\left[\frac{1}{1+\phi}+\hat{y}_{t}+\frac{1+\phi}{2} \hat{y}_{t}^{2}+\hat{\Delta}_{t}+\frac{1+\phi}{2} \hat{\Delta}_{t}^{2}\right] .
$$

The unconditional mean of the right-hand-side term of the utility function reads as:

$$
E\left[\frac{\chi}{1+\phi}\left(\Delta_{t} y_{t}\right)^{1+\phi}\right] \simeq \frac{\epsilon-1}{\epsilon}\left[\frac{1}{1+\phi}+\frac{1+\phi}{2}\left(E\left[\hat{y}_{t}^{2}\right]+E\left[\hat{\Delta}_{t}^{2}\right]\right)\right] .
$$

Gathering Eqs. (41) and (42), the unconditional mean of the utility function $\mathcal{U}(\cdot)$ as defined in Eq. (30) up to second order reads as:

$$
E[\mathcal{U}(\cdot)] \simeq-\frac{\epsilon-1}{\epsilon} \frac{1}{1+\phi}-\frac{\epsilon-1}{\epsilon}\left[\frac{1+\phi}{2} E\left[\hat{y}_{t}^{2}\right]+\frac{1+\phi}{2} E\left[\hat{\Delta}_{t}^{2}\right]\right] .
$$

It is straightforward to notice that the expression of the utility function includes the variance of the price dispersion $E\left[\hat{\Delta}_{t}^{2}\right]$. To obtain a closed-form expression of this variance, we perform a second-order approximation of the expression in Eq. 37:

$$
\begin{aligned}
1+\Delta_{t}+\frac{1}{2} \Delta_{t}^{2} \simeq(1-\theta)+\theta & \left(\hat{\pi}_{t}+\frac{1}{2} \hat{\pi}_{t}^{2}\right)+\frac{1}{2} \frac{\theta(2 \theta+(\epsilon-2))}{(1-\theta)} \hat{\pi}_{t}^{2} \\
& +\theta\left(1+\hat{\Delta}_{t-1}+\frac{1}{2} \hat{\Delta}_{t-1}^{2}+\epsilon\left(\hat{\pi}_{t}+\frac{1}{2} \hat{\pi}_{t}^{2}\right)+\frac{(\epsilon-1)}{2} \hat{\pi}_{t}^{2}\right) .
\end{aligned}
$$

The unconditional mean of the price dispersion allows us to obtain the expression of the variance of the price dispersion as:

$$
E\left[\Delta_{t}^{2}\right] \simeq \frac{\theta[\epsilon+2(1-\theta)(\epsilon-1)]}{(1-\theta)^{2}} E\left[\hat{\pi}_{t}^{2}\right]
$$

where $E\left[\Delta_{t}^{2}\right]=E\left[\Delta_{t-1}^{2}\right]$ and $E\left[\pi_{t}^{2}\right]=E\left[\pi_{t-1}^{2}\right]$, as the variance is unconditional. Notice 
that this expression is very close to the expression in Woodford (2002).

It can be shown that the first- and second-order derivatives of the price dispersion terms are given by:

$$
\left.\frac{\partial(1-\theta)^{\frac{\epsilon}{\epsilon-1}}\left(1-\theta \bar{\pi}^{1-\epsilon} \pi_{t-1}^{\epsilon-1}\right)^{1 /(1-\epsilon)}}{\partial \pi_{t-1}}\right|_{\pi_{t-1}=\bar{\pi}}=\left.\frac{\theta \bar{\pi}(1-\theta)^{\frac{\epsilon}{\epsilon-1}}\left(1-\theta \bar{\pi}^{1-\epsilon} \pi_{t-1}^{\epsilon-1}\right)^{\frac{1}{(1-\epsilon)}}}{\bar{\pi}^{\epsilon} \pi_{t-1}^{2-\epsilon}-\bar{\pi} \theta \pi_{t-1}}\right|_{\pi_{t-1}=\bar{\pi}}=\frac{\theta}{\bar{\pi}},
$$

and

$$
\begin{aligned}
&\left.\frac{\partial^{2}(1-\theta)^{\frac{\epsilon}{\epsilon-1}}\left(1-\theta \bar{\pi}^{1-\epsilon} \pi_{t-1}^{\epsilon-1}\right)^{1 /(1-\epsilon)}}{\partial^{2} \pi_{t-1}}\right|_{\pi_{t-1}=\bar{\pi}} \\
&=\left.\frac{\theta \bar{\pi}(1-\theta)^{\frac{\epsilon}{\epsilon-1}} \pi_{t-1}^{\epsilon-2}\left(1-\theta \bar{\pi}^{1-\epsilon} \pi_{t-1}^{\epsilon-1}\right)^{\frac{1}{(1-\epsilon)}}\left(2 \theta \bar{\pi} \pi_{t-1}^{\epsilon}+\bar{\pi}^{\epsilon} \pi_{t-1}(\epsilon-2)\right)}{\left(\bar{\pi}^{\epsilon} \pi_{t-1}-\theta \bar{\pi} \pi_{t-1}^{\epsilon}\right)^{2}}\right|_{\pi_{t-1}=\bar{\pi}}=\frac{\theta(2 \theta+\epsilon-2)}{\bar{\pi}^{2}(1-\theta)} .
\end{aligned}
$$

Replacing the price dispersion term given by Eq. (44), we obtain the final expression of the utility function up to a second order:

$$
E[\mathcal{U}(\cdot)] \simeq-\frac{\epsilon-1}{\epsilon} \frac{1}{1+\phi}-\frac{\epsilon-1}{\epsilon} \frac{1+\phi}{2}\left[E\left[\hat{y}_{t}^{2}\right]+\frac{\theta[\epsilon+2(1-\theta)(\epsilon-1)]}{(1-\theta)^{2}} E\left[\hat{\pi}_{t}^{2}\right]\right]
$$

The last step is to obtain the welfare index from the utility function. Recall that the welfare is defined by the discounted sum of future utility streams. Put recursively, the welfare index reads as:

$$
\mathcal{W}_{t}=\mathcal{U}\left(C_{t}, H_{t}\right)+\beta \mathcal{W}_{t+1}
$$

The unconditional mean of the welfare reads as:

$$
E\left[\mathcal{W}_{t}\right]=\frac{1}{1-\beta} E\left[\mathcal{U}\left(C_{t}, H_{t}\right)\right]
$$

as $E\left[\mathcal{W}_{t}\right]=E\left[\mathcal{W}_{t+1}\right]$. Finally, replacing the utility function into the previous expression gives:

$$
\begin{aligned}
E\left[\mathcal{W}_{t}\right] & \simeq-\frac{\epsilon-1}{\epsilon(1-\beta)} \frac{1}{1+\phi}-\frac{\epsilon-1}{\epsilon(1-\beta)} \frac{1+\phi}{2}\left[E\left[\hat{y}_{t}^{2}\right]+\frac{\theta[\epsilon+2(1-\theta)(\epsilon-1)]}{(1-\theta)^{2}} E\left[\hat{\pi}_{t}^{2}\right]\right] \\
& \equiv \overline{\mathcal{W}}-\lambda^{y} E\left[\hat{y}_{t}^{2}\right]-\lambda^{\pi} E\left[\hat{\pi}_{t}^{2}\right]
\end{aligned}
$$

with $\overline{\mathcal{W}} \equiv-\frac{\epsilon-1}{\epsilon(1-\beta)} \frac{1}{1+\phi}$ the steady-state level of welfare and $\lambda^{y} \equiv \frac{\epsilon-1}{\epsilon(1-\beta)} \frac{1+\phi}{2}$ and $\lambda^{\pi} \equiv$ $\lambda^{y} \frac{\theta[\epsilon+2(1-\theta)(\epsilon-1)]}{(1-\theta)^{2}}$ the elasticities, respectively, of the loss function with respect to the variance of output $E\left[\hat{y}_{t}^{2}\right]$ and inflation $E\left[\hat{\pi}_{t}^{2}\right]$. 


\section{D.4 Welfare cost}

Suppose now that we have two regimes, one with RE and one with SL expectations, and we want to measure the welfare cost of one regime with respect to the other. Following Lucas (2003), we look for the fraction $\lambda$ of utility that the representative household would be willing to pay to live under the RE regime rather than under the SL regime through the no-arbitrage condition on welfare indexes in both regimes:

$$
\sum_{t=0}^{\infty} \beta^{t} \mathcal{U}\left((1+\lambda) C_{t}^{R E}, H_{t}^{R E}\right)=\sum_{t=0}^{\infty} \beta^{t} \mathcal{U}\left(C_{t}^{S L}, H_{t}^{S L}\right)
$$

Approximating this expression up to a second order and using the relation in Eq. (48), the expression of the welfare cost between the two expectation regimes is given by:

$$
\lambda=\exp \left((1-\beta)\left(\lambda^{\pi}\left[\operatorname{var}\left(\hat{\pi}_{t}^{R E}\right)-\operatorname{var}\left(\hat{\pi}_{t}^{S L}\right)\right]+\lambda^{y}\left[\operatorname{var}\left(\hat{y}_{t}^{R E}\right)-\operatorname{var}\left(\hat{y}_{t}^{S L}\right)\right]+\right)\right)-1
$$

This is the metric that we use in the paper to compare outcomes of the model under the RE and the SL regimes and under the communication policies. 